\title{
The vanilloid receptor TRPV1 is activated and sensitized by local anesthetics in rodent sensory neurons
}

Andreas Leffler, ${ }^{1}$ Michael J. Fischer, ${ }^{2}$ Dietlinde Rehner, ${ }^{1}$ Stephanie Kienel, ${ }^{1}$ Katrin Kistner, ${ }^{2}$ Susanne K. Sauer, ${ }^{2}$ Narender R. Gavva, ${ }^{3}$ Peter W. Reeh, ${ }^{2}$ and Carla Nau ${ }^{1}$

1Department of Anesthesiology and 2Department of Physiology and Pathophysiology, Friedrich-Alexander-University Erlangen-Nuremberg, Erlangen, Germany. ${ }^{3}$ Department of Neuroscience, Amgen Inc., Thousand Oaks, California, USA.

\begin{abstract}
Local anesthetics (LAs) block the generation and propagation of action potentials by interacting with specific sites of voltage-gated $\mathrm{Na}^{+}$channels. LAs can also excite sensory neurons and be neurotoxic through mechanisms that are as yet undefined. Nonspecific cation channels of the transient receptor potential (TRP) channel family that are predominantly expressed by nociceptive sensory neurons render these neurons sensitive to a variety of insults. Here we demonstrated that the LA lidocaine activated TRP channel family receptors TRPV1 and, to a lesser extent, TRPA1 in rodent dorsal root ganglion sensory neurons as well as in HEK293t cells expressing TRPV1 or TRPA1. Lidocaine also induced a TRPV1-dependent release of calcitonin gene-related peptide (CGRP) from isolated skin and peripheral nerve. Lidocaine sensitivity of TRPV1 required segments of the putative vanilloid-binding domain within and adjacent to transmembrane domain 3 , was diminished under phosphatidylinositol 4,5-bisphosphate depletion, and was abrogated by a point mutation at residue R701 in the proximal C-terminal TRP domain. These data identify TRPV1 and TRPA1 as putative key elements of LA-induced nociceptor excitation. This effect is sufficient to release CGRP, a key component of neurogenic inflammation, and warrants investigation into the role of TRPV1 and TRPA1 in LA-induced neurotoxicity.
\end{abstract}

\section{Introduction}

Local anesthetics (LAs) block voltage-gated $\mathrm{Na}^{+}$channels, the major generators of the upstroke of action potentials $(1,2)$. The clinical use for spinal anesthesia and peripheral regional anesthesia requires the administration of highly concentrated LAs in order to achieve rapid and sufficient pain suppression $(3,4)$. Clinically used formulations of $1 \%-5 \%$ lidocaine correspond to about $40-200 \mathrm{mM}$ lidocaine. Neurotoxicity is a well-known side effect of highly concentrated LAs applied in the vicinity of neuronal structures (5). Several molecular mechanisms have been considered to mediate LA-induced cytotoxicity leading to cell death, including direct membrane disruption (6), mitochondrial dysfunction (7), and activation of the p38 mitogen-activated protein kinase involved in apoptosis $(8,9)$. Furthermore, LAs were previously demonstrated to directly depolarize sensory neurons by mediating an increase in intracellular $\mathrm{Ca}^{2+}\left(\left[\mathrm{Ca}^{2+}\right]_{i}\right)(10,11)$. The molecular transduction mechanism for this action of LAs is poorly understood. Current responses of cultured dorsal root ganglion (DRG) neurons to capsaicin and heat can be both enhanced and inhibited by LAs $(12,13)$. Capsaicin and noxious heat sensitivity of sensory neurons are mediated by the capsaicin receptor TRPV1 $(14,15)$. TRPV1 is a nonspecific cation channel of the transient receptor potential (TRP) channel family and is predominantly expressed by nociceptive sensory neurons $(16,17)$. As a polymodal receptor, TRPV1 ren-

Nonstandard abbreviations used: BCTC, N-(4-tertiarybutylphenyl)-4-(3-cholorpy-

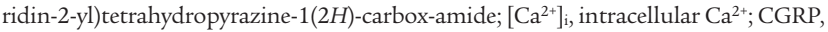
calcitonin gene-related peptide; DRG, dorsal root ganglion; LA, local anesthetic; o-, rabbit; $\mathrm{PAO}$, phenylarsine oxide; $\mathrm{PI}(4,5) \mathrm{P}_{2}$, phosphatidylinositol 4,5-bisphosphate; PMA, phorbol-12-myristate-13-acetate; $r-$, rat; TRP, transient receptor potential.

Conflict of interest: N.R. Gavva is employed by and owns equity in Amgen Inc. The remaining authors have declared that no conflict of interest exists.

Citation for this article: J. Clin. Invest. 118:763-776 (2008). doi:10.1172/JCI32751. ders sensory neurons sensitive to heat above $42^{\circ} \mathrm{C}$ and chemical irritants such as vanilloids, protons (16), ethanol (18), camphor (19), divalent cations (20), and polyamines (21).

Considering these features of TRPV1, we explored whether excitatory effects of LAs on sensory neurons are caused by a direct interaction with TRPV1 and other TRP channels. We found that LAs activated TRPV1 and - to a lesser extent - TRPA1 and identified what we believe to be a novel pathway by which LAs can activate and sensitize TRPV1. This action might contribute to modulation of peripheral nociception and to neurotoxicity.

\section{Results}

Lidocaine induces TRPV1-dependent $\left[\mathrm{Ca}^{2+}\right]_{i}$ rise and inward currents in DRG neurons. To test the hypothesis that lidocaine induces an increase in $\left[\mathrm{Ca}^{2+}\right]_{\mathrm{i}}$, DRG sensory neurons derived from adult rats were first explored using ratiometric $\mathrm{Ca}^{2+}$ measurements. In concentrations of $1 \mathrm{mM}$ or greater, lidocaine repeatedly evoked an increase in $\left[\mathrm{Ca}^{2+}\right]_{\mathrm{i}}$ in a concentration-dependent manner (Figure 1A). The corresponding $\mathrm{EC}_{50}$ value was $3.4 \pm 1.4 \mathrm{mM}$ (Figure $1 \mathrm{~B}$ ). The majority of cells investigated responded to lidocaine (118 of 129), and these cells were predominantly small and medium sized $(33.7 \pm 0.6 \mu \mathrm{m}$; Figure 1C). Strikingly, all but 1 of the capsaicin-sensitive cells also responded to lidocaine (107 of 108). In contrast, 11 of 21 capsaicininsensitive cells displayed a lidocaine-evoked increase of $\left[\mathrm{Ca}^{2+}\right]_{i}$. The comparably large fraction of capsaicin-sensitive cells compared with reported data $(30 \%-80 \%$, depending on the species and the peripheral origin of afferent fibers) might be due to some loss of largesized cells during the process of single-cell culture. The substantial overlap of lidocaine and capsaicin sensitivity suggests an involvement of TRPV1 in the lidocaine response of DRG neurons. This was corroborated by whole-cell voltage clamp experiments. As demonstrated in Figure 1D, 30 mM lidocaine evoked large inward cur- 
A
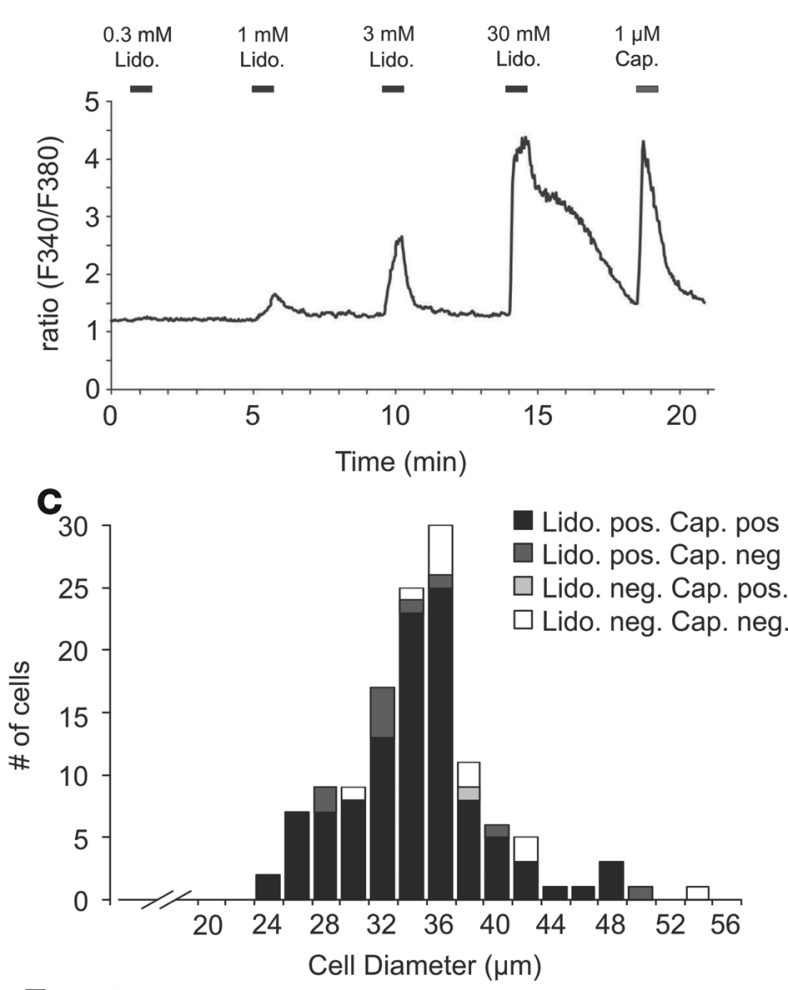

E Cap+

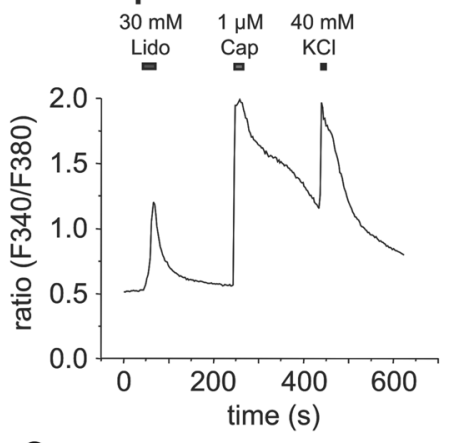

G

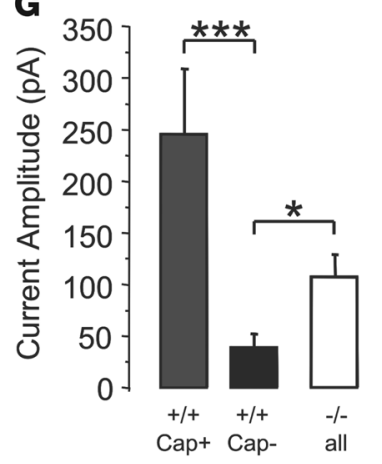

\section{Cap-}

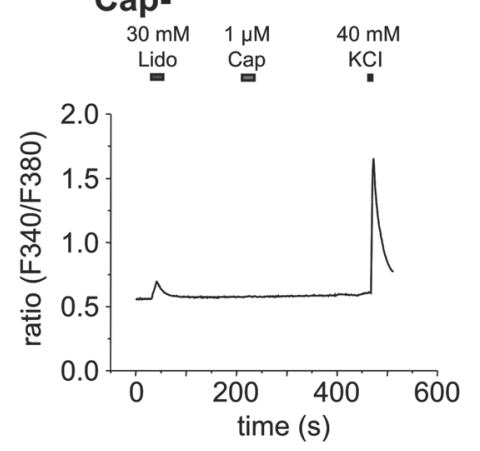

H WT

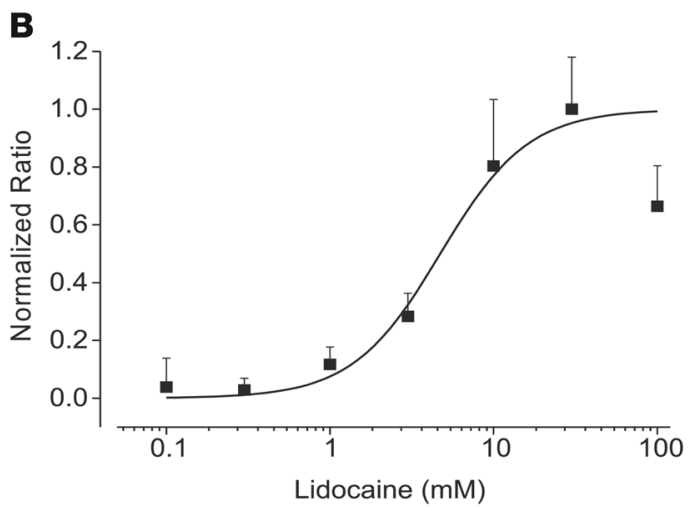

D

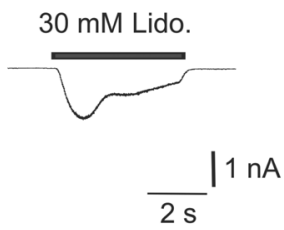

$1 \mu \mathrm{M}$ Cap.

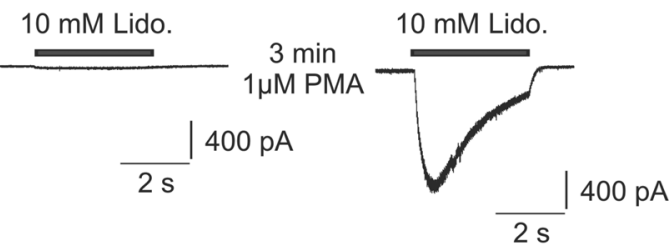

$\mathbf{F}$

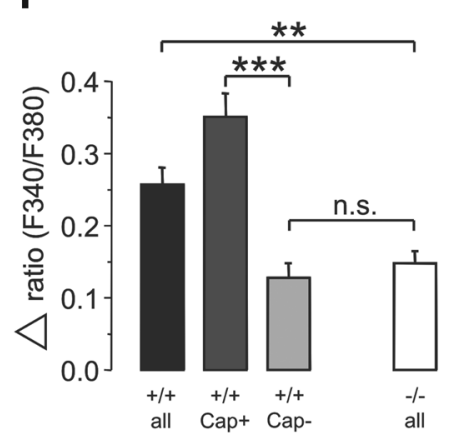

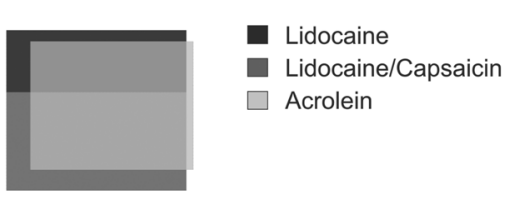

TRPV1-I-

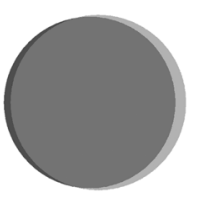

Lidocaine

Lidocaine/Acrolein

$\square$ Acrolein

\section{Figure 1}

Lidocaine induces an increase of $\left[\mathrm{Ca}^{2+}\right]_{i}$ and inward currents in DRG neurons. (A) Representative experiment showing increasing changes in fluorescence ratios F340/F380 with increasing concentrations of lidocaine in a capsaicin-sensitive rat DRG neuron. Cells were treated with increasing concentrations of lidocaine for $40 \mathrm{~s}$ in intervals of $4 \mathrm{~min}$. After lidocaine treatment, cells were subsequently treated with $1 \mu \mathrm{M}$ capsaicin. (B) Doseresponse curve for lidocaine-evoked $\left[\mathrm{Ca}^{2+}\right]_{\mathrm{i}}$ rise in rat DRG neurons. Due to an incomplete recovery of responses to 30 and $100 \mathrm{mM}, 2$ independent sets of experiments were used to compile the dose-response curve: $0.3,1,3$, and $30 \mathrm{mM}(n=65)$ and $0.1,3,10$, and 100 mM $(n=64)$. The data were fitted to the Hill equation. (C) Cell diameter of rat DRG neurons that were sensitive or insensitive to lidocaine and capsaicin. (D) Representative inward currents activated by lidocaine $(30 \mathrm{mM})$ and capsaicin $(1 \mu \mathrm{M})$ and the effect of PMA on lidocaine-evoked currents in rat 
DRG neurons. Cells were held at $-60 \mathrm{mV}$, and test solutions were applied in intervals of $3 \mathrm{~min}$. (E) Changes in fluorescence ratios F340/F380 in a capsaicin-sensitive and -insensitive mouse DRG neuron. Cells were treated with $30 \mathrm{mM}$ lidocaine ( $30 \mathrm{~s}), 1 \mu \mathrm{M}$ capsaicin (10 s), and $40 \mathrm{mM}$ $\mathrm{KCl}(10 \mathrm{~s})$ in intervals of 3 minutes. Only cells displaying a clear response to $\mathrm{KCl}$ were included in the analysis. (F) Mean changes in fluorescence ratios [ $\Delta$ ratio (F340/F380)] induced by $30 \mathrm{mM}$ lidocaine in mouse WT and TRPV1-- DRG neurons (unpaired Student's $t$ test). The designation "all" denotes all neurons of the respective genotype that were examined. WT neurons were divided into 2 categories, capsaicin sensitive (Cap $\left.{ }^{+}\right)$and insensitive (Cap-). (G) Mean current amplitudes evoked by $30 \mathrm{mM}$ lidocaine in mouse WT $(n=49)$ and TRPV1-/- $(n=35)$ neurons. WT neurons were divided into capsaicin-sensitive and -insensitive neurons. Both WT and TRPV1-/- neurons were divided into acrolein-sensitive (Acro+) and -insensitive (Acro-) neurons (unpaired Student's $t$ test). Neurons that neither responded to lidocaine $(30 \mathrm{mM})$, nor capsaicin $(1 \mu \mathrm{M})$, nor acrolein $(100 \mu \mathrm{M})(\mathrm{WT}, 12$ of 49; TRPV1-l-, 12 of 35) were included. (H) Area-proportional Venn diagram to represent the fraction of WT or TRPV1-/- neurons responding to 1, 2, or 3 of the substances lidocaine, capsaicin, and acrolein. Cells responding to none of the substances were excluded. ${ }^{\star} P<0.05 ;{ }^{* *} P<0.01 ;{ }^{* *} P<0.001$.

rents in 10 of 11 capsaicin-sensitive neurons $(1.4 \pm 0.4 \mathrm{nA}, n=10)$. The PKC-activator PMA strongly sensitized the response of DRG neurons to $10 \mathrm{mM}$ lidocaine ( $40.9 \pm 12.7$-fold, $n=3$ ), an effect comparable to PKC-mediated potentiation of TRPV1 gating by capsaicin, protons, or heat (22). To confirm the role of TRPV1 in mediating the lidocaine-evoked increase of $\left[\mathrm{Ca}^{2+}\right]_{i}$ in DRG neurons, we used DRG neurons of WT and TRPV1 $1^{-/-}$mice. Capsaicin-sensitive neurons (WT, 44 of 76; TRPV1-/-, 0 of 89) displayed significantly larger lidocaine-evoked responses compared with capsaicin-insensitive neurons (WT, 32 of 76; TRPV1 $1^{--}, 89$ of 89; Figure 1, E and F). TRPV1 $1^{-/-}$neurons displayed an increase in $\left[\mathrm{Ca}^{2+}\right]_{\mathrm{i}}$ as well. However, this response was significantly smaller compared with capsaicinsensitive WT neurons and similar to capsaicin-insensitive WT neurons (Figure 1F). Measuring lidocaine-evoked currents in WT and TRPV1 $1^{-/}$neurons by means of whole-cell voltage clamp gave rise to similar results. Capsaicin-sensitive WT neurons (22 of 49) displayed significantly larger lidocaine-evoked currents than did capsaicininsensitive neurons (27 of 49) and TRPV1 ${ }^{-/-}$neurons (Figure 1G). To further clarify the effect of lidocaine in capsaicin-insensitive and TRPV1 $1^{-/-}$neurons, we tested the lidocaine sensitivity of several TRP channels heterologously expressed in HEK293t cells and found that in addition to TRPV1 channels, TRPA1 channels were activated by lidocaine (Supplemental Figure 1A; supplemental material available online with this article; doi:10.1172/JCI32751DS1), whereas TRPV2, TRPV3, TRPV4, and TRPM8 channels were not. TRPA1 is an excitatory ion channel that is selectively expressed by a subset of unmyelinated, peptidergic nociceptors that also express TRPV1. TRPA 1 is activated by pungent irritants from mustard and garlic and by environmental irritants such as acrolein (23). We found that both WT and TRPV1 $1^{-/-}$neurons that responded to acrolein also exhibited significant lidocaine-evoked currents. More importantly, acrolein-insensitive TRPV1-/- neurons and acrolein- and capsaicin-insensitive WT neurons did not show any significant lidocaine-evoked currents (Figure $1 \mathrm{G}$ ). With respect to the fraction of cells responding to 1, 2, or 3 of the substances lidocaine, capsaicin, and acrolein, we found that the predominant fraction of lidocainesensitive but capsaicin-insensitive WT neurons exhibited acrolein sensitivity (Figure 1H). Accordingly, nearly all of the lidocaine-sensitive TRPV1 $1^{-1-}$ neurons exhibited acrolein sensitivity (Figure $1 \mathrm{H}$ ). These data demonstrate that TRPV1 and TRPA1 are activated by lidocaine. As the lidocaine-evoked increase in $\left[\mathrm{Ca}^{2+}\right]_{i}$ and the lidocaine-evoked current amplitudes were significantly larger in capsaicin-sensitive neurons, the excitatory effects of LAs on sensory neurons might be predominantly mediated by TRPV1.

Extracellularly applied lidocaine requires a bydrophobic pathway to activate and sensitize recombinant TRPV1. To study the effect of lidocaine on TRPV1 in more detail, HEK293t cells transiently expressing rat TRPV1 (rTRPV1) were examined with the whole-cell voltage- clamp technique. As demonstrated in Figure 2A, lidocaine activated inward currents in concentrations of $3 \mathrm{mM}$ or greater when cells were held at $-60 \mathrm{mV}$. The mean peak current amplitudes at $3 \mathrm{mM}(178 \pm 92 \mathrm{pA}, n=18), 10 \mathrm{mM}(1,022 \pm 288 \mathrm{pA}, n=11), 30 \mathrm{mM}$ $(10.5 \pm 1.6 \mathrm{nA}, n=11)$, and $100 \mathrm{mM}(9.9 \pm 1.2 \mathrm{nA}, n=11)$ suggest a steep concentration dependence of lidocaine-evoked currents, yielding an $\mathrm{EC}_{50}$ of about $12 \mathrm{mM}$ (Figure $2 \mathrm{~B}$ ). At a concentration of $100 \mathrm{mM}$, lidocaine consistently activated a rapidly inactivating current. Frequently, the leak current subsequently increased, probably reflecting a seal break induced by $100 \mathrm{mM}$ lidocaine. In mocktransfected cells, lidocaine in concentrations up to $100 \mathrm{mM}$ did not evoke any significant currents. Again, an increase in leak current was observed in 3 of 6 cells following application of $100 \mathrm{mM}$ lidocaine (data not shown). When cells were held at $+60 \mathrm{mV}$, lidocaine evoked outward currents with distinct properties compared with inward currents. As demonstrated in Figure 2C, the outward currents were accompanied by rapidly deactivating tail currents upon washout of lidocaine $(n=5)$. When $500 \mathrm{~ms}$ lasting voltage-ramps from -100 to $+100 \mathrm{mV}$ were applied, TRPV1 exhibited outward rectification in the presence of both $30 \mathrm{mM}$ lidocaine and $30 \mathrm{nM}$ capsaicin (Figure 2D). The tail currents, as evoked upon washout of lidocaine at $+60 \mathrm{mV}$, suggest that lidocaine might also block the channel pore of TRPV1. To test this hypothesis, the effect of lidocaine on currents activated by a saturating concentration of capsaicin $(10 \mu \mathrm{M})$ was investigated at $-60 \mathrm{mV}$ and $+60 \mathrm{mV}$. Indeed, lidocaine blocked both inward and outward currents in a concentration-dependent and reversible manner (Figure 2, E and F).

To further corroborate the hypothesis that lidocaine specifically activates TRPV1, we examined the effect of well-known and wellcharacterized TRPV1 antagonists on lidocaine-evoked currents. As demonstrated in Figure 2, G-I, lidocaine-evoked currents were almost completely blocked by the noncompetitive antagonist ruthenium red, a blocker of several TRP channels ( $5 \mu \mathrm{M} ; 97.2 \% \pm 0.6 \%$ block, $n=7)$, by the competitive TRPV1 antagonist capsazepine $(10 \mu \mathrm{M} ; 86.7 \% \pm 1.4 \%$ block, $n=7)$, and by the highly selective TRPV1 antagonist N-(4-tertiarybutylphenyl)-4-(3-cholorpyridin-2-yl)tetrahydropyrazine-1(2H)carbox-amide (BCTC; $100 \mathrm{nM} ; 98.5 \% \pm 0.2 \%$ block, $n=6$ ).

We also examined the effect of several other LAs on TRPV1. Amide-type LAs including mepivacaine, articaine, and bupivacaine as well as ester-type LAs including procaine and tetracaine activated TRPV1 (Supplemental Figure 2, A-E).

A hallmark feature of TRPV1 is the ability to integrate multiple activating stimuli when applied simultaneously, leading to a reciprocal cross-sensitization (17). In order to investigate whether lidocaine sensitizes TRPV1 currents, $3 \mathrm{mM}$ lidocaine was applied while TRPV1 currents were evoked by various stimuli. At $3 \mathrm{mM}$, lidocaine potentiated TRPV1 currents evoked by thermal stimuli (6.7 \pm 1.2 -fold, $n=7$; Figure $3 \mathrm{~A}$ ), by $30 \mathrm{nM}$ capsaicin $(3.7 \pm 0.9$ - 
A $1 \mathrm{mM}$ Lido. $3 \mathrm{mM}$ Lido. $10 \mathrm{mM}$ Lido. $30 \mathrm{mM}$ Lido. $100 \mathrm{mM}$ Lido.
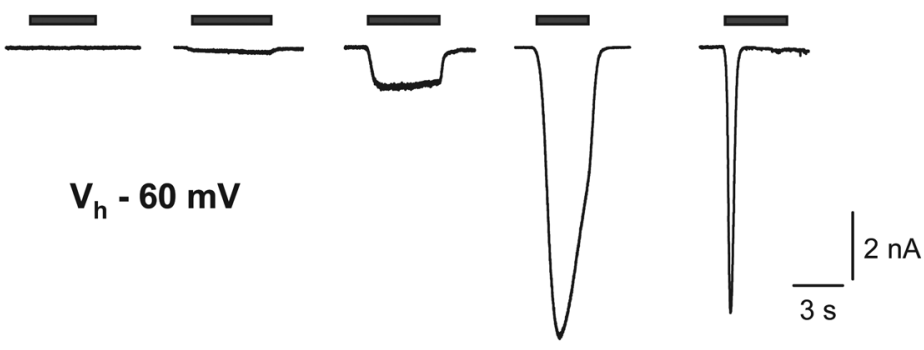

C

$3 \mathrm{mM}$ Lido. $10 \mathrm{mM}$ Lido. $\quad 30 \mathrm{mM}$ Lido.

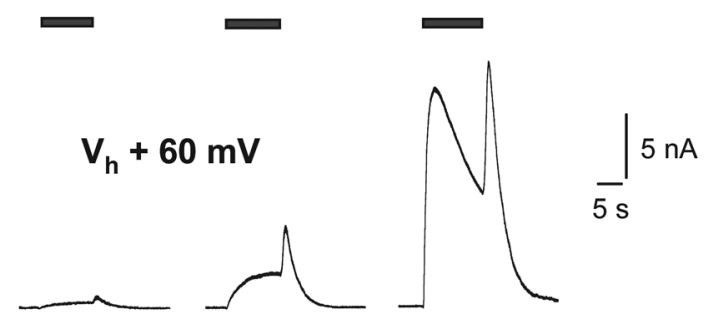

B

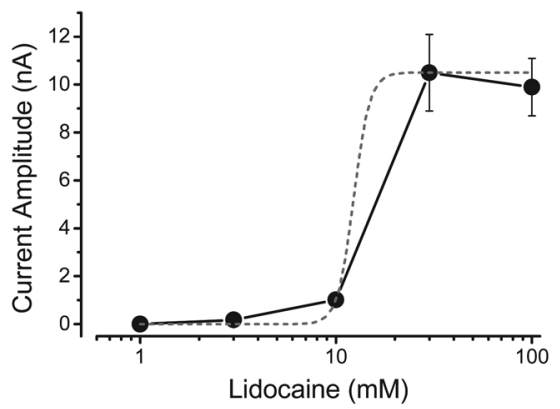

D
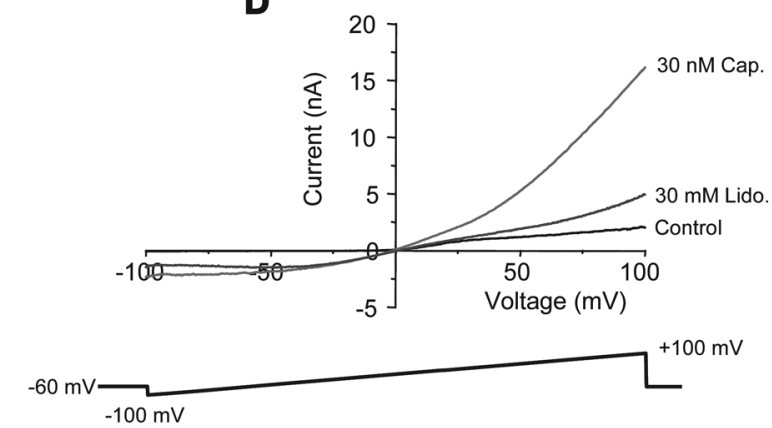

E
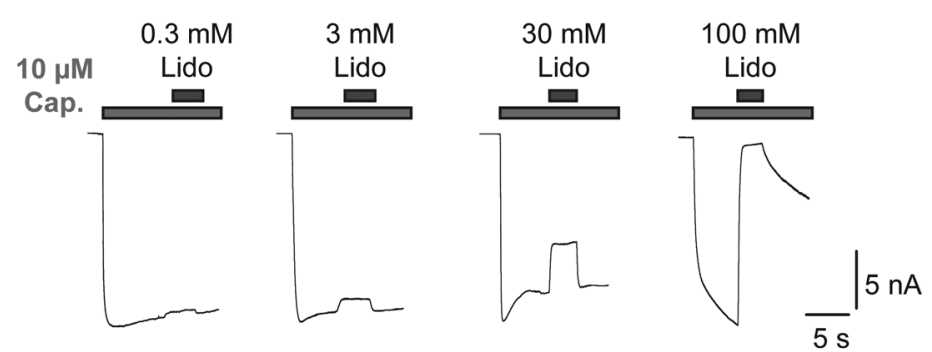

\section{$\mathbf{F}$}

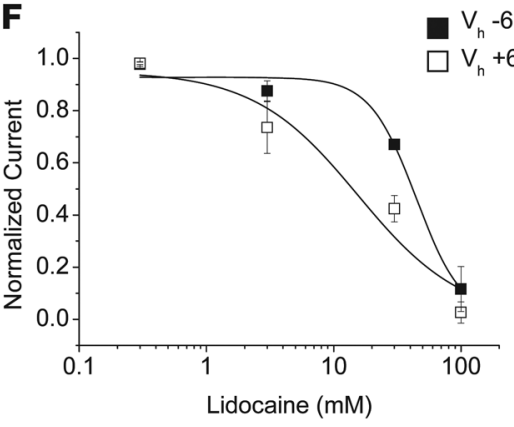

G $5 \mu \mathrm{M} R \mathrm{R}$

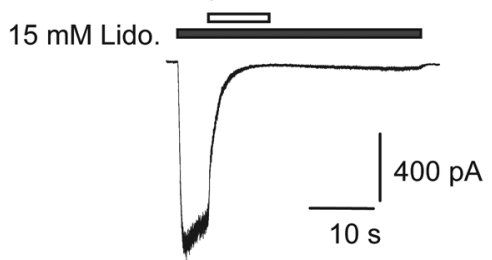

H

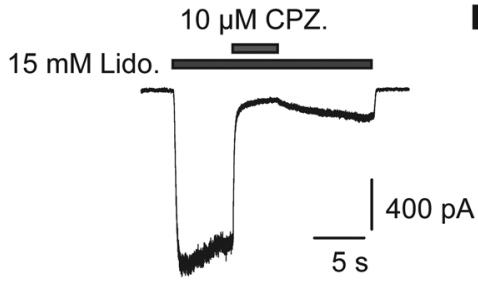

I

$100 \mathrm{nM}$ BCTC

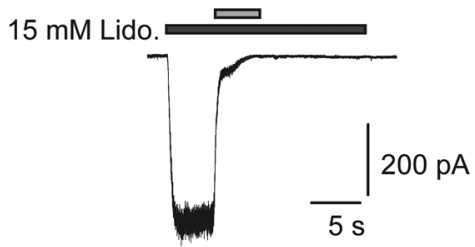

Figure 2

Lidocaine activates and blocks recombinant rTRPV1 expressed in HEK293t cells. (A) Representative responses to 1, 3, 10, 30, and 100 mM lidocaine of HEK293t cells transiently expressing rTRPV1. The holding potential $\left(\mathrm{V}_{h}\right)$ was $-60 \mathrm{mV}$. Various concentrations of lidocaine were applied for about $10 \mathrm{~s}$. Only 1 concentration per cell was applied. (B) Concentration-response curve for lidocaine. Each data point represents mean peak current amplitudes evoked by 1, 3, 10,30, or $100 \mathrm{mM}$ lidocaine in 11 independent cells each. The line represents a fit of the data to the Hill equation. (C) Outward currents evoked by 3,10 , and $30 \mathrm{mM}$ lidocaine in a HEK293t cell held at $+60 \mathrm{mV}$. Lidocaine was applied for about $10 \mathrm{~s}$ in intervals of $2 \mathrm{~min}$. (D) Representative ramp currents of TRPV1 in control solution, in $30 \mathrm{mM}$ lidocaine, and in $30 \mathrm{nM}$ capsaicin $(n=8)$. Cells were held at $-60 \mathrm{mV}$, and currents were measured during $500-\mathrm{ms}$-long voltage ramps from -100 to $+100 \mathrm{mV}$. Note the typical outward rectification of TRPV1 at $30 \mathrm{mM}$ lidocaine and $30 \mathrm{nM}$ capsaicin. (E) Representative capsaicin-evoked inward currents blocked by lidocaine. Increasing concentrations of lidocaine were coapplied with capsaicin after currents were evoked by a supramaximal concentration of capsaicin $(10 \mu \mathrm{M})$. Cells were held at $-60 \mathrm{mV}$, and experiments were performed in a $\mathrm{Ca}^{2+}$-free extracellular solution in order to avoid desensitization. (F) Dose-response curve for lidocaine-induced block of TRPV1 inward $\left(\mathrm{V}_{\mathrm{h}},-60 \mathrm{mV}\right)$ and outward $\left(\mathrm{V}_{\mathrm{h}},+60 \mathrm{mV}\right)$ currents evoked by capsaicin. The fractional block was plotted against the lidocaine concentration. Lines represent fits of the data to the Hill equation. The $I_{50}$ values were $45 \pm 7 \mathrm{mM}(n=4)$ at $-60 \mathrm{mV}$ and $16 \pm 17 \mathrm{mM}(n=4)$ at $+60 \mathrm{mV}$. (G-I) Effect of the noncompetitive TRPV1 antagonist ruthenium red (RR, $5 \mu \mathrm{M}$; G), the competitive TRPV1 antagonist capsazepine (CPZ, $10 \mu \mathrm{M} ; \mathbf{H})$, and the highly selective TRPV1 antagonist BCTC (100 nM; I) on lidocaine-evoked currents in HEK293t cells expressing TRPV1. The substances were coapplied with 15 mM lidocaine after currents evoked by $15 \mathrm{mM}$ lidocaine had reached steady state. 


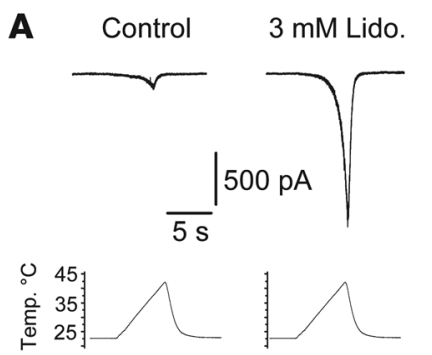

D

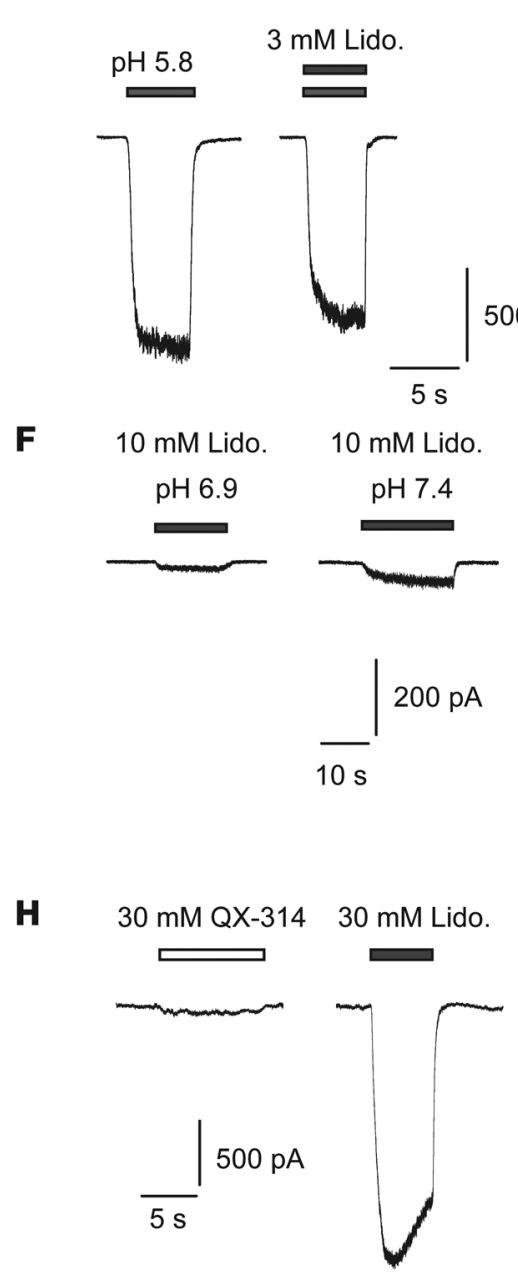

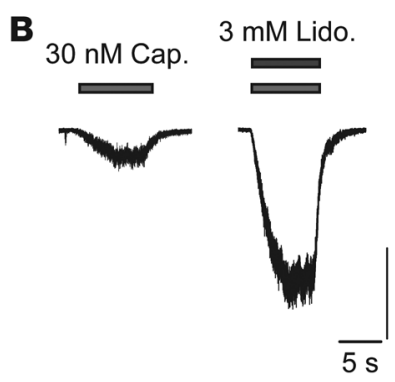

C

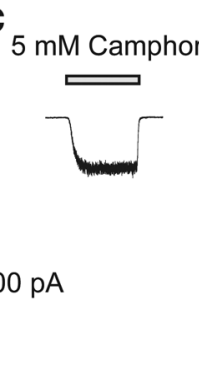

E
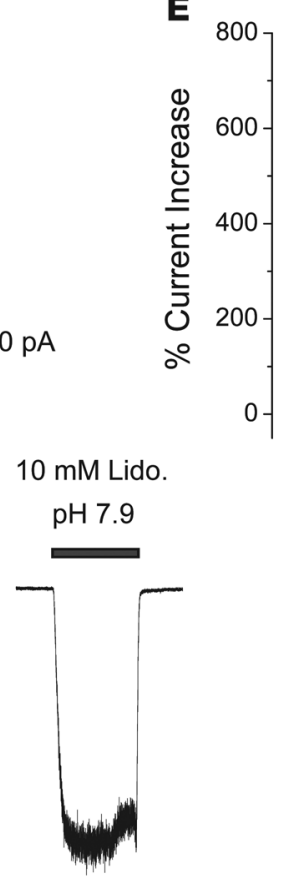

I

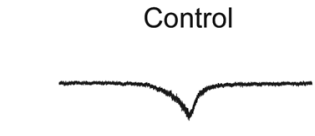

pH $6.9 \mathrm{pH} 7.4 \mathrm{pH} 7.9$

$3 \mathrm{mM}$ QX-314
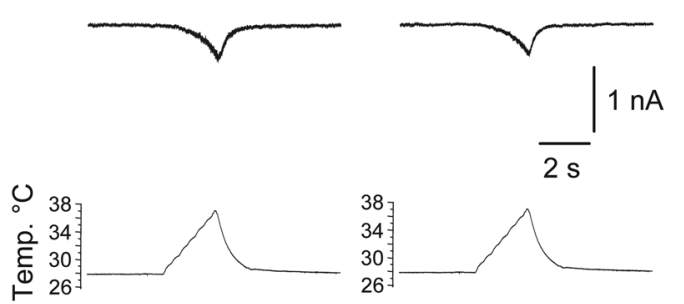

Figure 3

Lidocaine sensitizes and activates TRPV1 in a pH-dependent manner. (A) Representative heatevoked inward currents of TRPV1 in control solution and in the presence of $3 \mathrm{mM}$ lidocaine. Currents were evoked by repeated thermal stimuli consisting of a 8-s-long linear rise of temperature from $23^{\circ} \mathrm{C}$ to $42^{\circ} \mathrm{C}$. (B-D) Effect of 3 $\mathrm{mM}$ lidocaine on TRPV1 currents activated by $30 \mathrm{nM}$ capsaicin (B), $5 \mathrm{mM}$ camphor (C), or protons (pH 5.8; D). (E) Mean increase of inward currents (percentage \pm SEM) measured in experiments described in $\mathbf{A}-\mathbf{D}$. In $\mathbf{A}-\mathbf{D}$, experiments were performed in $\mathrm{Ca}^{2+}$ free extracellular solution and cells were held at $-60 \mathrm{mV}$. (F) Representative TRPV1 currents evoked by $10 \mathrm{mM}$ lidocaine at $\mathrm{pH}$ 6.9, 7.4, and 7.9. Test solutions with increasing $\mathrm{pH}$ values were applied in intervals of $2 \mathrm{~min}$. Control solution with $\mathrm{pH} 7.4$ and no lidocaine was applied in between. (G) Mean current amplitudes \pm SEM measured as described in $\mathbf{F}$ and normalized to values obtained at $\mathrm{pH} 7.4$ (paired Student's $t$ test). (H) Effect of the membraneimpermeable lidocaine derivative QX-314 compared with lidocaine on TRPV1 when applied extracellularly. Cells were held at $-60 \mathrm{mV}$, and $30 \mathrm{mM} \mathrm{QX}-314$ and $30 \mathrm{mM}$ lidocaine were applied for about $10 \mathrm{~s}$ in intervals of $2 \mathrm{~min}$. (I) Heatevoked inward currents of TRPV1 in control solution and in the presence of $3 \mathrm{mM} \mathrm{QX-314.} \mathrm{Currents}$ were evoked by repeated thermal stimuli as described in A. Note that QX-314 neither activated nor sensitized TRPV1 when applied extracellularly. ${ }^{\star \star \star} P<0.001$. fold, $n=9$; Figure $3 \mathrm{~B})$, or by $5 \mathrm{mM}$ camphor $(3.2 \pm 0.3$-fold, $n=4$; Figure $3 \mathrm{C}$ ). Comparable with lidocaine, articaine and procaine also potentiated TRPV1 currents evoked by thermal stimuli $(7.0 \pm 1.2$ fold, $n=7$, and $5.3 \pm 0.4$-fold, $n=6$, respectively; Supplemental Figure $2, \mathrm{~F}$ and $\mathrm{G}$ ) and evoked an increase in $\left[\mathrm{Ca}^{2+}\right]_{\mathrm{i}}$ in DRG neurons (Supplemental Figure 2H). These findings support the idea that LAs as a class may have TRPV1-activating properties.

At a concentration of $3 \mathrm{mM}$, lidocaine failed to potentiate TRPV1 currents activated by pH 5.8 (0.94 \pm 0.08 -fold, $n=10$; Figure $3 \mathrm{D})$; $100 \mathrm{mM}$ lidocaine, however, potentiated those currents $(2.4 \pm 0.5$ fold, $n=5$; data not shown). It is important to note that lidocaine is a weak base with a $\mathrm{pK}_{\mathrm{a}}$ value of 7.9. The fraction of nonionized, membrane-permeable lidocaine molecules is about 0.25 at $\mathrm{pH} 7.4$ and about 0.008 at $\mathrm{pH}$ 5.8. When applying $10 \mathrm{mM}$ lidocaine at $\mathrm{pH} 6.9$ (membrane-permeable fraction, 0.1), $\mathrm{pH} 7.4$, and pH 7.9 (membrane-permeable fraction, 0.5 ; Figure $3 \mathrm{~F}$ ), the currents evoked at $\mathrm{pH} 6.9$ were significantly smaller $(0.5 \pm 0.05$-fold, $n=10)$ and the currents evoked at $\mathrm{pH} 7.9$ were significantly larger (10 \pm 1.1 -fold, $n=10$ ) compared with the currents evoked at $\mathrm{pH} 7.4$ (Figure $3 \mathrm{G}$ ). These data suggest that the membrane-permeable rather than the ionized fraction of lidocaine is required for activation of TRPV1. The possibility that protonated lidocaine activates TRPV1 via interaction with residues regulating proton sensitivity of TRPV1 was ruled out by the finding that the proton-insensitive mutants TRPV1-E600A and TRPV1-E648A (24) generated large currents when treated with $30 \mathrm{mM}$ lidocaine (Supplemental Figure 3, A-C). Moreover, the membrane-impermeable, quaternary derivative of lidocaine QX-314 (30 mM) neither activated TRPV1 nor 


\section{Table 1}

Absolute and relative current responses of rat and rabbit WT and mutant channels to lidocaine, capsaicin, and protons

\begin{tabular}{lccccc} 
& \multicolumn{2}{c}{ Mean current amplitude $(\mathbf{n A})$} & \multicolumn{2}{c}{ Normalized current } \\
Construct & Lidocaine $\mathbf{( 3 0} \mathbf{~ m M )}$ & Capsaicin $\mathbf{( 1 0} \boldsymbol{\mu M} \mathbf{M})$ & Protons $\mathbf{( p H ~ 5 . 0 )}$ & Lidocaine/capsaicin & Lidocaine/protons \\
rTRPV1-WT & $3.0 \pm 1.0(\mathbf{1 9})$ & $12.9 \pm 5.9(9)$ & $6.0 \pm 0.14(10)$ & $0.12 \pm 0.08(7)$ & $0.5 \pm 0.07(10)$ \\
rTRPV1-Y511A & $0.92 \pm 0.21(7)$ & $0.28 \pm 0.14(7)$ & $3.9 \pm 1.1(7)$ & $7.9 \pm 2.0(7)$ & $0.4 \pm 0.09(7)$ \\
OTRPV1-WT & $0.08 \pm 0.02(7)$ & $1.4 \pm 0.5(7)$ & $1.9 \pm 0.8(7)$ & $0.08 \pm 0.02(7)$ & $0.09 \pm 0.03(7)$ \\
OTRPV1-I550T & $0.22 \pm 0.05(13)$ & $10.6 \pm 2.3(6)$ & $5.8 \pm 1.6(7)$ & $0.02 \pm 0.01(6)$ & $0.06 \pm 0.02(7)$ \\
r/0 chimera & $2.3 \pm 0.7(20)$ & $7.2 \pm 1.1(10)$ & $6.3 \pm 2.4(10)$ & $0.18 \pm 0.04(10)$ & $0.6 \pm 0.09(10)$ \\
\hline
\end{tabular}

the legend to Figure 4. Numbers in parentheses denote $n$.

potentiated heat-evoked TRPV1 currents $(0.93 \pm 0.08$-fold, $n=5)$ when applied extracellularly (Figure 3, H and I). However, $30 \mathrm{mM}$ QX-314 was more potent than $30 \mathrm{mM}$ lidocaine to exert the presumptive pore-blocking effect, inhibiting capsaicin-evoked inward currents, whereas lidocaine was more potent to inhibit capsaicinevoked outward currents (Supplemental Figure 3, D-F).

Activation, but not sensitization, of TRPV1 by lidocaine involves the vanilloid-binding domain. Both capsaicin and capsazepine have been suggested to interact with a vanilloid-binding domain composed of transmembrane domains 3 and 4 and the respective cytosolic interfaces $(25,26)$. To explore whether lidocaine activates TRPV1 through interaction with the vanilloid binding site, we investigated the action of lidocaine in the rabbit TRPV1 homolog (oTRPV1), which is sensitive to protons and heat but 100 -fold less sensitive to vanilloids than rTRPV1 (26). The relative responsiveness of oTRPV1 and rTRPV1 to $30 \mathrm{mM}$ lidocaine and $10 \mu \mathrm{M}$ capsaicin or protons ( $\mathrm{pH}$ 5.0) was examined (Table 1). Both lidocaine- and capsaicin-activated currents were small in oTRPV1 (Figure 4A). However, the ratio of lidocaine- to capsaicin-evoked currents was similar in oTRPV1 and rTRPV1 (Figure 4C). In contrast, the ratio of lidocaine- to proton-evoked currents was significantly smaller in oTRPV1 than in rTRPV1 (Figure 4, B and D). These data indicate that the low capsaicin sensitivity of oTRPV1 is accompanied by a low lidocaine sensitivity. To further narrow down the region of TRPV1 that is required for activation by lidocaine, mutant constructs of rTRPV1 and oTRPV1 were used. The point mutation rTRPV1-Y511A has been shown to abrogate vanilloid sensitivity (25). Surprisingly, rTRPV1-Y511A displayed some lidocaine-evoked currents, so that the ratio of lidocaine- to capsaicin-evoked currents was significantly larger than in WT rTRPV1 (Figure 4C). The ratio of lidocaine- to proton-evoked currents for rTRPV1-Y511A was similar to that of WT rTRPV1 (Figure 4D). Thus mutation Y511A results in a loss of capsaicin but not in a complete loss of lidocaine sensitivity. Both the point mutation oTRPV1-I550T and the r/o chimera, in which transmembrane domains TM3 through TM4 (amino acids S505-T550) of rTRPV1 were transferred to oTRPV1, confer vanilloid sensitivity to oTRPV1 (26). As expected, oTRPV1-I550T generated significantly larger capsaicin-evoked currents compared with oTRPV1-WT $(10.6 \pm 2.3 \mathrm{nA}, n=6$, versus $1.4 \pm 0.5 \mathrm{nA}, n=7)$. However, the ratio of lidocaine- to capsaicinevoked currents was significantly smaller and the ratio of lidocaineto proton-evoked currents was similar compared with oTRPV1-WT (Figure 4, C and D). Thus, mutation I550T of oTRPV1 results in a significant gain of capsaicin but an insignificant gain of lidocaine sensitivity. In the r/o chimera, the ratio of lidocaine- to capsaicin-evoked currents was similar to both oTRPV1-WT and rTRPV1-WT (Figure 4C). The ratio of lidocaine- to proton-evoked currents was significantly larger compared with oTRPV1-WT but similar to rTRPV1 (Figure 4D). Thus the transfer of the vanilloidbinding domain from rTRPV1 to oTRPV1 results in a gain of both vanilloid and lidocaine sensitivity, which suggests that activation of TRPV1 by lidocaine indeed involves part of the vanilloid-binding domain. Intriguingly, $3 \mathrm{mM}$ lidocaine still potentiated capsaicin- $(2.9 \pm 0.4$-fold, $n=5)$ and heat-evoked (3.4 \pm 1.5 -fold, $n=6)$ currents in oTRPV1-WT (Figure 4, E and F). These data suggest that the mechanism of sensitization by lidocaine does not depend on the vanilloid binding site and might be disparate from that of activation. We also probed the sensitivity of human TRPV1 toward lidocaine and found that human TRPV1 generated large currents when treated with $30 \mathrm{mM}$ lidocaine (Supplemental Figure 4).

Different mechanisms underlie tachyphylaxis induced by lidocaine and capsaicin. A typical feature of TRPV1 is a pronounced desensitization and tachyphylaxis when activated repeatedly by agonists such as capsaicin and protons. This process is induced in part by a rise in $\left[\mathrm{Ca}^{2+}\right]_{i}$ that ultimately leads to dephosphorylation of TRPV1. Another mechanism of $\mathrm{Ca}^{2+}$ negative feedback and $\mathrm{Ca}^{2+}$-dependent inactivation involves the $\mathrm{Ca}^{2+}$ sensor calmodulin, which directly interacts with TRPV1 $(27,28)$. Desensitization is significantly reduced in the absence of extracellular $\mathrm{Ca}^{2+}$ ions (29). Comparable with other TRPV1 activators, $30 \mathrm{mM}$ lidocaine induced acute desensitization and tachyphylaxis when applied consecutively in $\mathrm{Ca}^{2+}$-containing extracellular solution (Figure 5A). Remarkably, desensitization and tachyphylaxis were even more pronounced when $\mathrm{Ca}^{2+}$ was removed from the extracellular solution (Figure 5C). Moreover, capsaicin $(1 \mu \mathrm{M})$ activated TRPV1 that had been desensitized by lidocaine in both $\mathrm{Ca}^{2+}$-containing and $\mathrm{Ca}^{2+}$-free extracellular solution (data not shown). Thus, lidocaine-induced desensitization and tachyphylaxis does not result in cross-desensitization for capsaicin. We reasoned that lidocaine-induced tachyphylaxis might be caused, at least in part, by a loss of signaling molecules due to cell dialysis. Indeed, lidocaine-activated currents were significantly smaller in cells examined 20 minutes after the whole-cell configuration had been established compared with cells examined after 2 minutes. No such rundown was observed for capsaicin-evoked currents (Figure 5G).

Phosphatidylinositol 4,5-bisphosphate, but not $P K C$, is required for activation and sensitization of TRPV1 by lidocaine. A candidate signaling pathway mediating activation of TRPV1 by lidocaine might involve stimulation of PLC and subsequent depletion of phosphatidylinositol 4,5-bisphosphate $\left[\mathrm{PI}(4,5) \mathrm{P}_{2}\right]$. Indeed, pretreatment of TRPV1- 
A rTRPV1

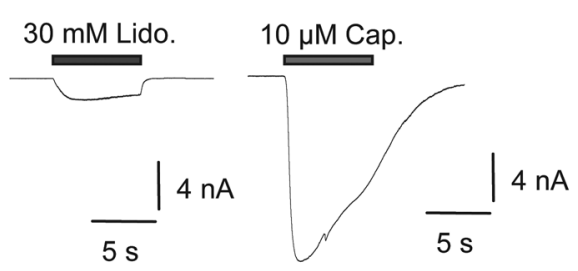

OTRPV1
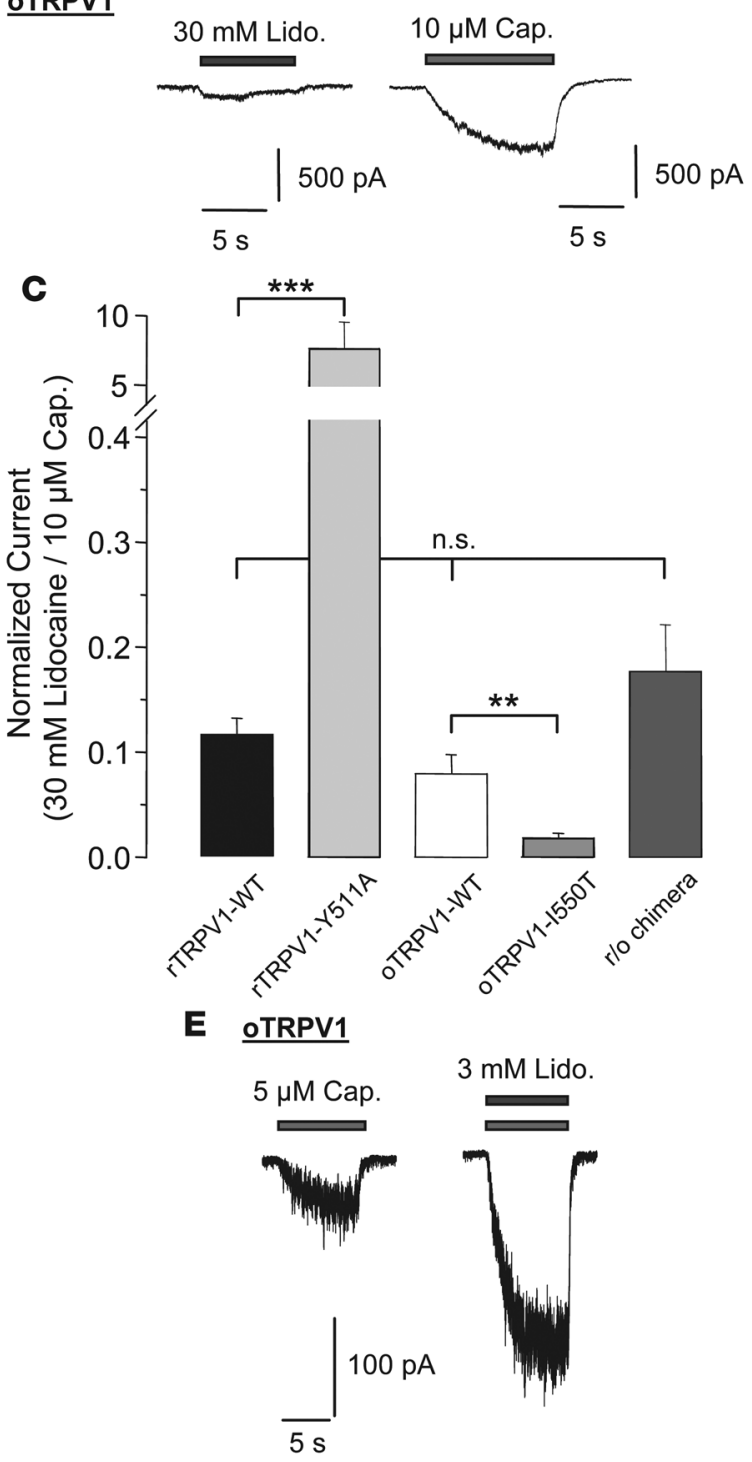

B $\underline{\text { rTRPV1 }}$

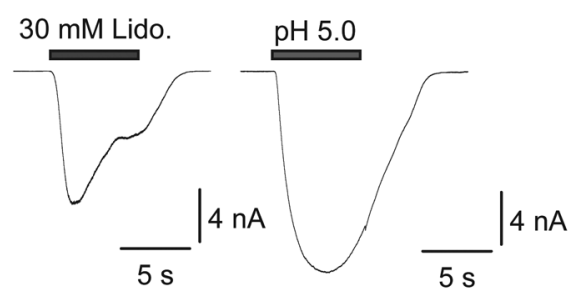

OTRPV1

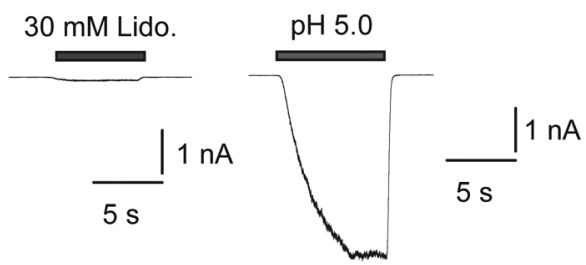

D

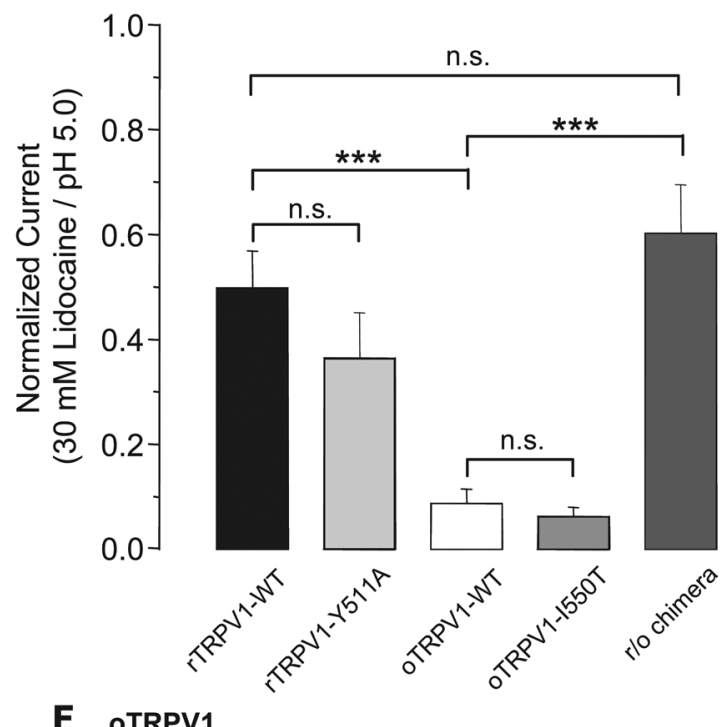

F oTRPV1
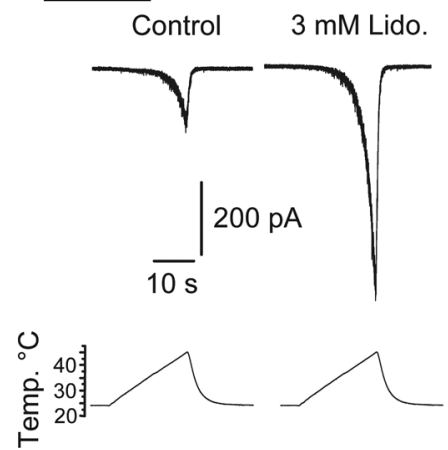

Figure 4

Activation, but not sensitization, of TRPV1 by lidocaine depends on the vanilloid-binding domain. (A and B) Representative currents of rTRPV1 and oTRPV1 activated by $30 \mathrm{mM}$ lidocaine and $10 \mu \mathrm{M}$ capsaicin $(\mathbf{A})$ or $30 \mathrm{mM}$ lidocaine and protons (pH 5.0; B). Each cell was treated with lidocaine followed by either capsaicin or protons. Cells were held at $-60 \mathrm{mV}$, and test solutions were applied every $2 \mathrm{~min}$. (C) Mean ratios of lidocaine- (30 mM) to capsaicin-activated $(10 \mu \mathrm{M})$ currents of rTRPV1-WT, rTRPV1-Y511A, oTRPV1-WT, oTRPV1-550T, and r/oTRPV1 chimera. (D) Mean ratios of lidocaine- $(30 \mathrm{mM})$ to proton-activated ( $\mathrm{pH} \mathrm{5.0)}$ currents of rTRPV1 and oTRPV1 constructs. In C and D, statistical comparisons were calculated with unpaired Student's $t$ test; $n=5-8$ cells of each construct. (E and F) Effect of 3 mM lidocaine on capsaicin-evoked (E) and heat-evoked oTRPV1 currents (F). Experiments were performed as described for Figure $2 .{ }^{* \star} P<0.01 ;{ }^{* \star *} P<0.001$.

expressing HEK293t cells with the PLC blocker U73122, but not with the inactive analog U73433, resulted in a significant reduction of lidocaine- but not capsaicin-evoked currents (Supplemental
Figure 5). Unfortunately, U73122 also induced a slowly activating TRPV1 current and sensitized TRPV1 to capsaicin (Supplemental Figure 5). Thus we cannot rule out that desensitization of TRPV1, 


\section{A $\underline{2} \mathrm{mMCa}^{++}$}
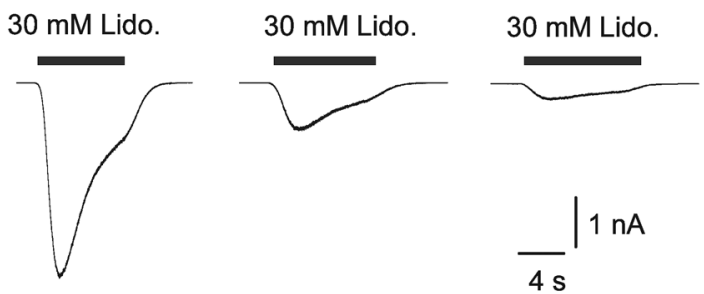

\section{C $\underline{\mathrm{Ca}^{++}-\text {free }}$}
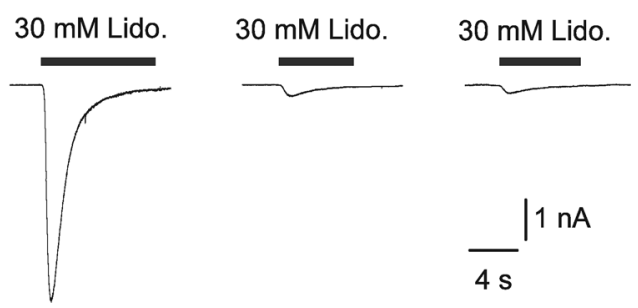

E

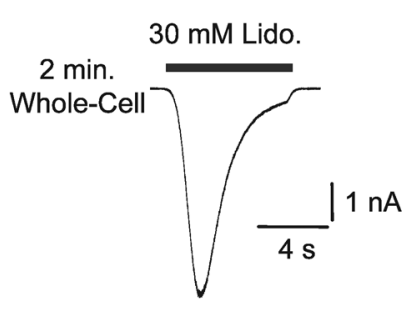

B

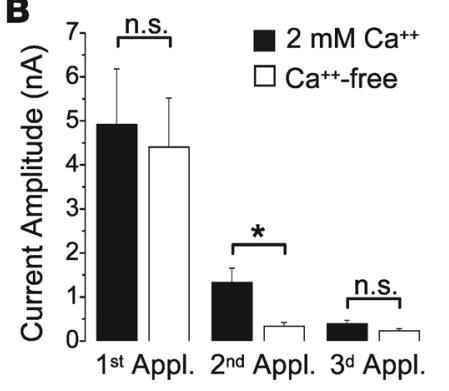

D
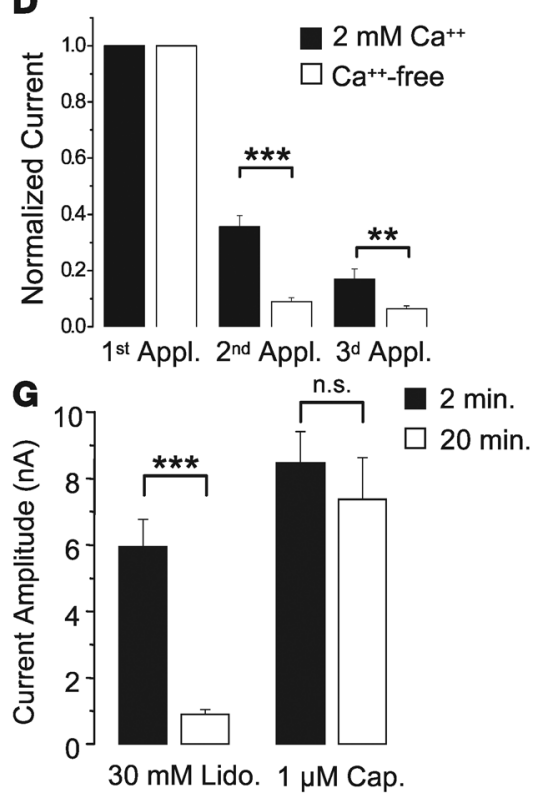

\section{Figure 5}

Lidocaine induces tachyphylaxis of TRPV1. (A and C) Representative TRPV1 currents activated by 3 consecutive applications of $30 \mathrm{mM}$ lidocaine in standard extracellular solution containing $2 \mathrm{mM} \mathrm{Ca}^{2+}(\mathbf{A} ; n=12)$ or in $\mathrm{Ca}^{2+-f r e e ~ e x t r a c e l l u l a r ~ s o l u-~}$ tion in which $2 \mathrm{mM} \mathrm{CaCl}_{2}$ was replaced by 5 mM EGTA (C; $n=8$ ). Lidocaine was applied in intervals of $2 \mathrm{~min}$. (B and D) Mean current amplitudes \pm SEM measured in experiments described in $\mathbf{A}$ and $\mathbf{C}$. In D, current amplitudes are normalized to the value obtained with the first application of lidocaine (unpaired Student's $t$ test). (E and F) Representative lidocaine-evoked inward currents activated 2 minutes (E) or 20 minutes (F) after whole-cell mode was established. (G) Mean current amplitudes \pm SEM measured in experiments described in $\mathbf{E}$ and $\mathbf{F}$ with $30 \mathrm{mM}$ lidocaine or with $1 \mu \mathrm{M}$ capsaicin ( $n=10$; unpaired Student's $t$ test). ${ }^{*} P<0.05 ;{ }^{* *} P<0.01$; ${ }^{* * *} P<0.001$. rather than inhibition of PLC, contributes to the inhibition of lidocaine-activated TRPV1 currents by U73122. PLC hydrolyzes $\mathrm{PI}(4,5) \mathrm{P}_{2}$ to generate diacylglycerol and inositol 1,4,5-triphosphate. The diacylglycerol analog 1-oleoyl-2-acetyl-glycerol (100-500 $\mu \mathrm{M})$ did not have any effect on TRPV1 (data not shown), suggesting that diacylglycerol is not involved in TRPV1 activation.

By inhibiting phosphatidylinositol 4-kinases, the replenishment of the membrane with $\mathrm{PI}(4,5) \mathrm{P}_{2}$ can be retarded, which also results in depletion of $\mathrm{PI}(4,5) \mathrm{P}_{2}$. In cells pretreated with the phosphatidylinositol 4-kinase blocker phenylarsine oxide (PAO; $30 \mu \mathrm{M}$ ) before whole-cell mode was established, amplitudes of lidocaine-activated currents were significantly reduced compared with untreated cells (Figure 6, A-C). PAO treatment also resulted in a significant reduction of capsaicin-evoked currents (Figure 6C), and, most remarkably, almost completely abolished the sensitizing effect of lidocaine on capsaicin- (0.96 \pm 0.07 -fold, $n=12)$ and heat-evoked (1.49 \pm 0.16 -fold, $n=8)$ currents (Figure 6, D and E). $\mathrm{PI}(4,5) \mathrm{P}_{2}$ was previously suggested to bind to the distal $\mathrm{C}$ terminus of TRPV1 to inhibit channel activity (30). We found, however, that a mutation lacking the distal C-terminus (TRPV1- $\Delta 767-838$ ) including the putative $\mathrm{PI}(4,5) \mathrm{P}_{2}$ binding region (residues $777-820$ ) did not affect the ability of lidocaine to activate or sensitize TRPV1 (Supplemental Figure 6). There are several recent reports on $\mathrm{PI}(4,5) \mathrm{P}_{2}$ regulating the activity of members of the TRP family by interacting with conserved positively charged residues in the highly conserved proximal C-terminal TRP domain (31). We therefore gen- erated the TRPV1 mutations K698Q, R701Q, and K710Q, in which these residues are replaced by neutral ones. Mutations K698Q $(5.3 \pm 1.3 \mathrm{nA}, n=7)$ and K710Q $(3.7 \pm 0.7 \mathrm{nA}, n=5)$, but not R701Q $(116 \pm 36 \mathrm{pA}, n=14)$, displayed large lidocaine-evoked currents (Figure 7, A-D). Moreover, capsaicin-evoked currents were strongly sensitized by $3 \mathrm{mM}$ lidocaine in K698Q (3.8 \pm 0.9 -fold, $n=5)$ and K710Q ( $3.4 \pm 0.8$-fold, $n=7)$, but not in R701Q ( $0.8 \pm 0.05$-fold, $n=7$; Figure $7, \mathrm{E}-\mathrm{H})$. A similar pattern was observed for heatevoked currents (data not shown). Thus, the properties of R701Q reflect those of TRPV1-WT treated with PAO.

$\mathrm{PKC}$ is another downstream second messenger of PLC activation. PKC is known to sensitize TRPV1 by phosphorylation of residues S502 and S800 (32, 33). However, phosphorylation by PKC was not crucial for lidocaine-evoked activation and sensitization of TRPV1, as both effects in a double mutation at putative PKC phosphorylation sites S502A and S800A were indistinguishable from TRPV1-WT (Supplemental Figure 7). Treatment of cells with the PKC activator phorbol-12-myristate-13-acetate (PMA; $1 \mu \mathrm{M}$ ) strongly potentiated lidocaine-evoked TRPV1-WT currents. This potentiation was abolished in the double mutation at S502A and S800A (Supplemental Figure 7).

LAs induce a TRPV1-dependent release of calcitonin gene-related peptide in isolated mouse skin and peripheral nerve. Activation of TRPV1 leads to a $\mathrm{Ca}^{2+}$-dependent release of proinflammatory peptides from primary afferent nerves such as the neuropeptide calcitonin generelated peptide (CGRP). CGRP contributes to neurogenic inflam- 

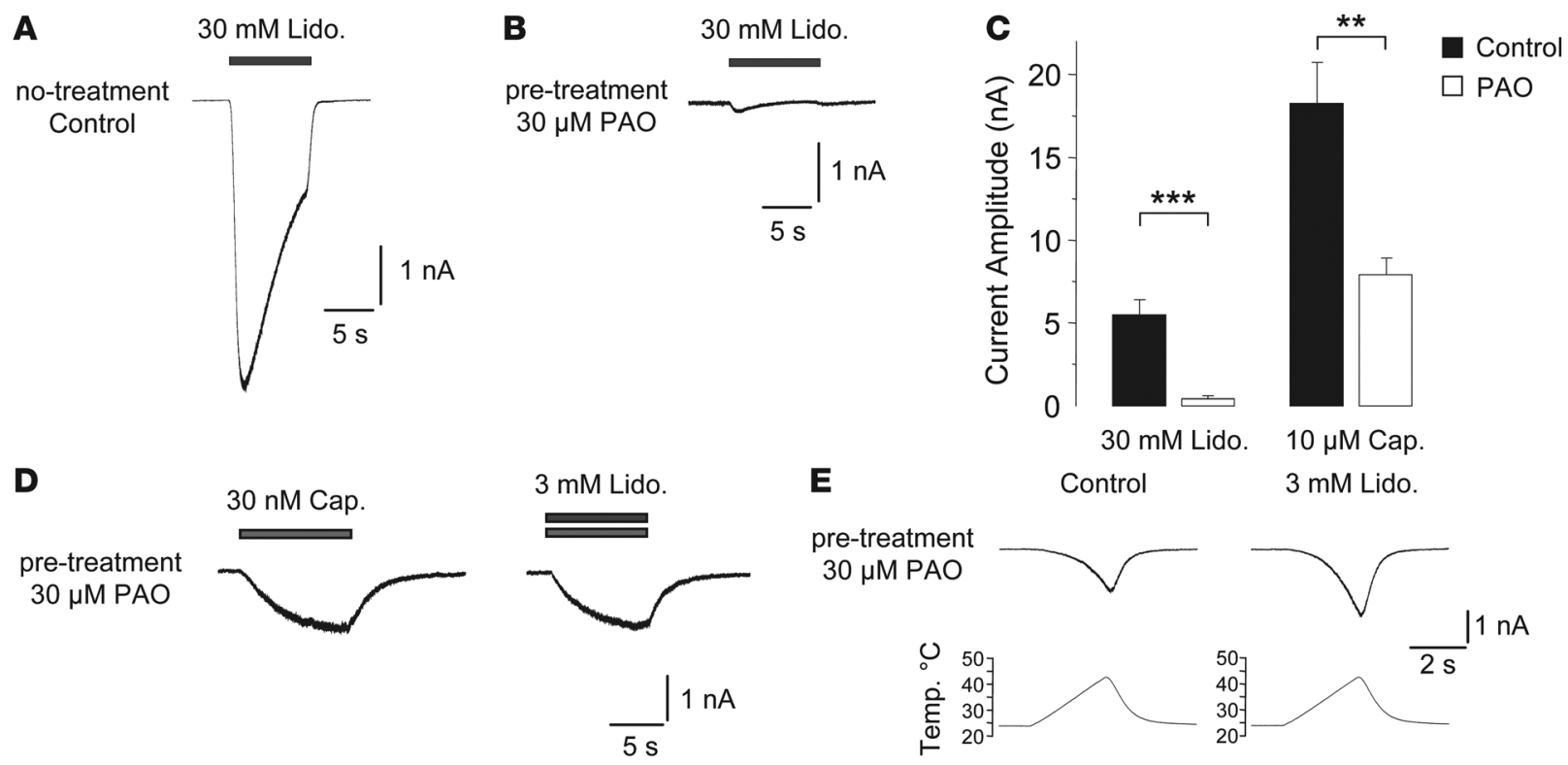

\section{Figure 6}

Lidocaine sensitivity of TRPV1 requires $\mathrm{PI}(4,5) \mathrm{P}_{2}$. (A and B) Representative lidocaine-evoked inward currents activated in HEK293t cells treated with control solution (A) or with the phosphatidylinositol 4-kinase blocker PAO (B) for 20 min before whole cell mode was established. (C) Mean current amplitudes \pm SEM measured in experiments described in $\mathbf{A}$ and $\mathbf{B}$ with $30 \mathrm{mM}$ lidocaine $(n=7)$ or with $10 \mu \mathrm{M}$ capsaicin $(n=8)$ (unpaired Student's $t$ test). (D and E) Typical effect of $3 \mathrm{mM}$ lidocaine on capsaicin-evoked (D) and heat-evoked (E) currents in HEK293t cells expressing TRPV1-WT treated with $30 \mu \mathrm{M}$ PAO before whole cell mode was established. ${ }^{\star *} P<0.01 ;{ }^{* \star} P<0.001$.

mation and to peripheral and central sensitization (34-36). In order to investigate whether the effect of lidocaine on TRPV1 is sufficient to evoke release of neuropeptides in native tissues, CGRP release was measured from hairy skin treated with lidocaine. As demonstrated in Figure 8A, lidocaine evoked a concentration-dependent CGRP release in skin from WT mice treated with 1,3 , and $30 \mathrm{mM}$ lidocaine. The role of TRPV1 in this process was demonstrated in skin derived from TRPV1-/- mice, which displayed a strongly attenuated CGRP release compared with WT when treated with $30 \mathrm{mM}$ lidocaine (Figure 8A). Mimicking the clinical use of LAs to block peripheral nerves, CGRP release was also measured from isolated sciatic nerves of WT and TRPV1 $1^{-1-}$ mice. While $30 \mathrm{mM}$ lidocaine induced CGRP release in both WT and TRPV1 $1^{-1-}$ nerves, the release in the latter was significantly smaller (Figure 8B). Coapplication of $10 \mu \mathrm{M}$ capsazepine effectively blocked CGRP release in WT nerves (Figure $8 \mathrm{~B})$. The amide-type LA articaine $(30 \mathrm{mM})$ and the ester-type LA procaine ( $30 \mathrm{mM}$ ) also evoked a substantial release of CGRP from WT sciatic nerve, similar to lidocaine (Figure 8B).

\section{Discussion}

TRPV1 has been attributed a central role in peripheral nociceptive transduction and is therefore being extensively studied. We show here that LAs activated and sensitized TRPV1 and thus add one more to the growing list of compounds that gate TRPV1. Our results not only describe what we believe to be a novel mechanism of activation of TRPV1 but also might provide a conceivable molecular explanation for side effects and sequelae induced by LAs when used for spinal and regional anesthesia.

Mechanism of lidocaine-induced $\left[\mathrm{Ca}^{2+}\right]_{i}$ rise in DRG neurons. Lidocaine induced a rapid increase in $\left[\mathrm{Ca}^{2+}\right]_{i}$ in both rat and mouse DRG neurons and evoked currents in both DRG neurons and HEK293t cells transiently expressing TRPV1. There is no doubt that a predominant fraction of current activated by lidocaine is mediated by TRPV1, as lidocaine did not evoke any significant currents in mock-transfected cells and the TRPV1 antagonists ruthenium red, capsazepine, and BCTC significantly blocked lidocaine-activated currents. There is also substantial evidence that a significant amount of the increase in $\left[\mathrm{Ca}^{2+}\right]_{\mathrm{i}}$ in DRG neurons is mediated by TRPV1: in rats, almost all capsaicin-sensitive neurons responded to lidocaine, and capsaicin-sensitive native neurons in mice exhibited $\left[\mathrm{Ca}^{2+}\right]_{i}$ increase significantly greater than that in capsaicin-insensitive or $T R P V 1^{-1-}$ neurons. However, $30 \mathrm{mM}$ lidocaine still evoked a significant increase in $\left[\mathrm{Ca}^{2+}\right]_{\mathrm{i}}$ and inward currents in capsaicin-insensitive $T R P V 1^{-/-}$neurons and evoked some CGRP release from skin and nerves harvested from TRPV1 $1^{-/}$mice. By directly testing the effect of lidocaine on other members of the TRP channel family, we found that TRPA $1-a$ channel coexpressed with TRPV1 in a subset of unmyelinated, peptidergic nociceptors - is also activated by lidocaine. The following observations point to TRPA1 as the mediator of the TRPV1-independent increase in $\left[\mathrm{Ca}^{2+}\right]_{\mathrm{i}}$ and inward currents: (a) a predominant fraction of lidocaine-sensitive but capsaicin-insensitive WT neurons exhibited sensitivity to acrolein, an activator of TRPA1; and (b) nearly all of the lidocaine-sensitive TRPV1 $1^{-/-}$neurons exhibited acrolein sensitivity. However, we cannot exclude that lidocaine may additionally evoke a release of $\mathrm{Ca}^{2+}$ from internal stores $(11,37)$.

Mechanisms of lidocaine-induced activation and sensitization of TRPV1. A dual molecular mechanism seems to underlie the activation of TRPV1 by LAs. LAs interact with a domain that is similar, but not identical, to the vanilloid-binding domain composed of transmembrane domains 3 and 4 and the respective cytosolic interfaces $(25,26)$. Additionally, activation of TRPV1 by LAs appeared to involve PLC and $\mathrm{PI}(4,5) \mathrm{P}_{2}$ signaling. The following observations 
A

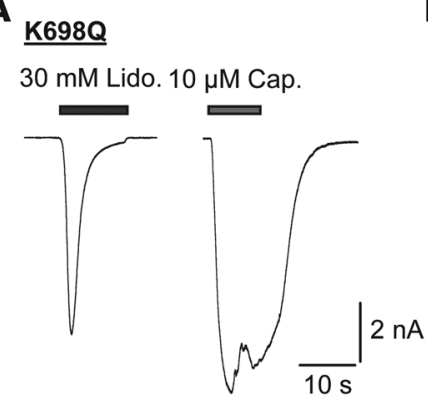

mM Lido. 10 uM Cap.

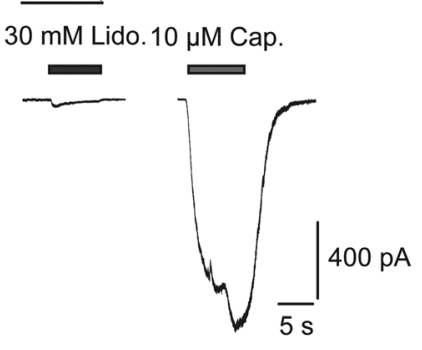

C

\section{$\underline{K 710 Q}$}

30 mM Lido. 10 M Cap.

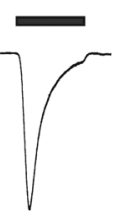

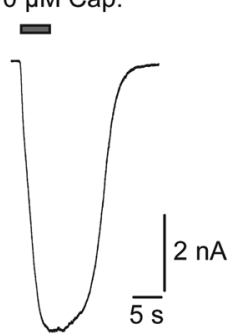

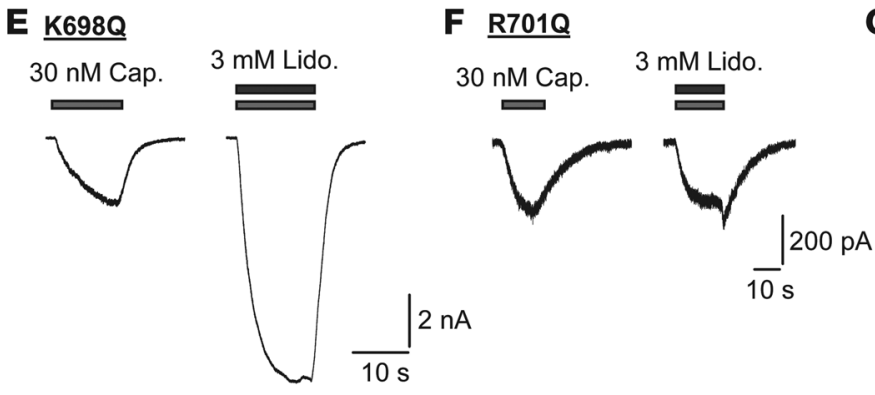

\section{G $\underline{\mathrm{K} 710 Q}$}

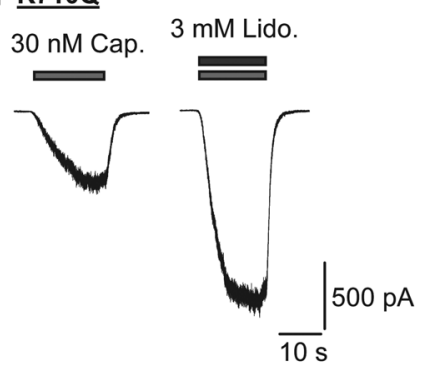

D
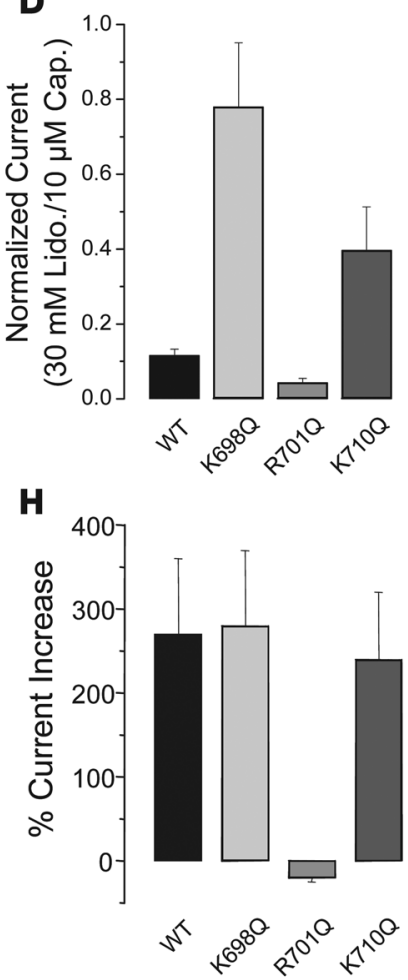

Figure 7

Interaction of $\mathrm{PI}(4,5) \mathrm{P}_{2}$ with positively charged residues within the proximal $\mathrm{C}$ terminus regulates the effect of lidocaine on TRPV1. (A-C) Representative current traces of the TRPV1 mutants K698Q (A), R701Q (B), and K710Q (C) activated by $30 \mathrm{mM}$ lidocaine and $10 \mu \mathrm{M}$ capsaicin. Cells were held at $-60 \mathrm{mV}$, and test solutions were applied every $2 \mathrm{~min}$. (D) Mean ratios of lidocaine- $(30 \mathrm{mM})$ to capsaicin-activated currents of the TRPV1 constructs WT, K698Q $(n=7), \mathrm{R} 701 \mathrm{Q}(n=14)$, and K710Q $(n=4)$. (E-G) Typical effect of 3 mM lidocaine on capsaicin-evoked currents in the TRPV1 mutants K698Q (E), R701Q (F), and K710Q (G). In E-G, experiments were performed as described in Figure 2. (H) Mean increase of heat-evoked inward currents in the presence of $3 \mathrm{mM}$ lidocaine (percentage \pm SEM) measured in experiments described in $\mathbf{E}-\mathbf{G}$.

suggest LA interaction with a molecular region similar to the vanilloid-binding domain: (a) a hydrophobic pathway was required for LA-induced activation; (b) the competitive TRPV1 antagonist capsazepine prevented activation of TRPV1 by lidocaine; and (c) lidocaine only weakly activated the vanilloid-insensitive oTRPV1 (86\% identical and 91\% similar to rTRPV1), but it strongly activated the vanilloid-sensitive $r$ /oTRPV1 chimera in which amino acids S505-T550 of rTRPV1 were transferred to oTRPV1.

Other data demonstrate that further regions of the channel might be involved in LA (and vanilloid) binding as well and that the binding domains for vanilloids and LAs are distinct. First, lidocaine and capsaicin produced small but detectable currents in oTRPV1, and, more importantly, lidocaine enhanced capsaicinand heat-activated oTRPV1 currents. A similar phenomenon has previously been described for capsaicin, which mildly enhanced proton-activated currents in the vanilloid-insensitive chicken TRPV1 (25). Second, lidocaine evoked insignificant currents in oTRPV1-I550T, which confers vanilloid sensitivity to oTRPV1 (26). Third, lidocaine activated the point-mutated rTRPV1-Y511A, which lacks significant vanilloid sensitivity.

We also found similarities and differences between the effect of camphor and LAs on TRPV1. Camphor is a lipophilic TRPV1 agonist that activates and sensitizes TRPV1. Similar to our findings with lidocaine, camphor failed to activate the vanilloid-insensitive chicken TRPV1 but was active on the capsaicin-insensitive point- mutant rTRPV1-Y511A (19). In contrast to the action of lidocaine, camphor-evoked rTRPV1 currents were not blocked by capsazepine; in addition, chicken TRPV1 did not gain camphor sensitivity by insertion of the rTRPV1 vanilloid binding domain (19). Camphor also activated TRPV3, which we found to be insensitive toward LAs (data not shown). The effect of camphor was strongly potentiated by lidocaine.

Comparable with other TRPV1 activators, lidocaine induced strong acute desensitization when applied for a prolonged time period and tachyphylaxis when applied consecutively. Desensitization and tachyphylaxis are typical features of TRPV1 that are induced, at least in part, by a rise in $\left[\mathrm{Ca}^{2+}\right]_{\mathrm{i}}$ that leads to channel dephosphorylation by mechanisms involving calcineurin (38). Desensitization of TRPV1 can be reduced by PKA- and PKC-mediated phosphorylation $(29,39,40)$. Another mechanism of $\mathrm{Ca}^{2+}$ negative feedback and $\mathrm{Ca}^{2+}$-dependent inactivation involves the $\mathrm{Ca}^{2+}$ sensor calmodulin $(27,28)$. Recovery from desensitization requires replenishment of the membrane with $\mathrm{PI}(4,5) \mathrm{P}_{2}$, which can be depleted upon activation (41). In contrast to capsaicin-induced desensitization, lidocaine-induced desensitization was retained after the removal of external $\mathrm{Ca}^{2+}$, which suggests an underlying mechanism that is $\mathrm{Ca}^{2+}$ independent. A similarly strong $\mathrm{Ca}^{2+}$-independent desensitization has previously been described for camphor (19). Camphor also dramatically enhanced tachyphylaxis of proton- and capsaicin-activated currents. Cells treated with $30 \mathrm{mM}$ 
A

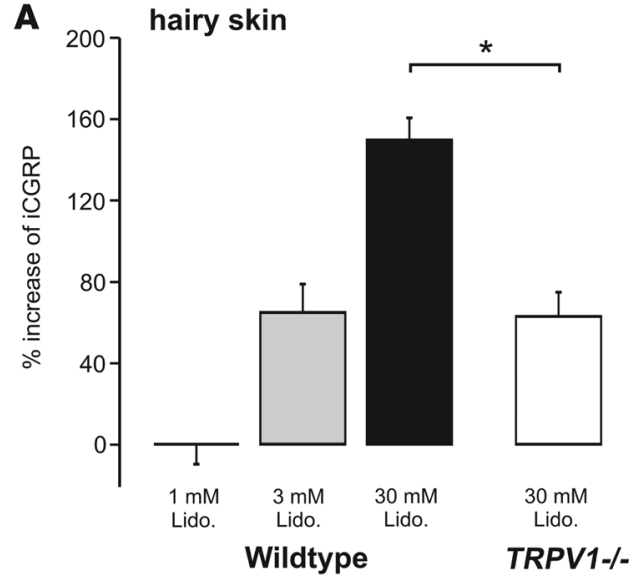

B sciatic nerve

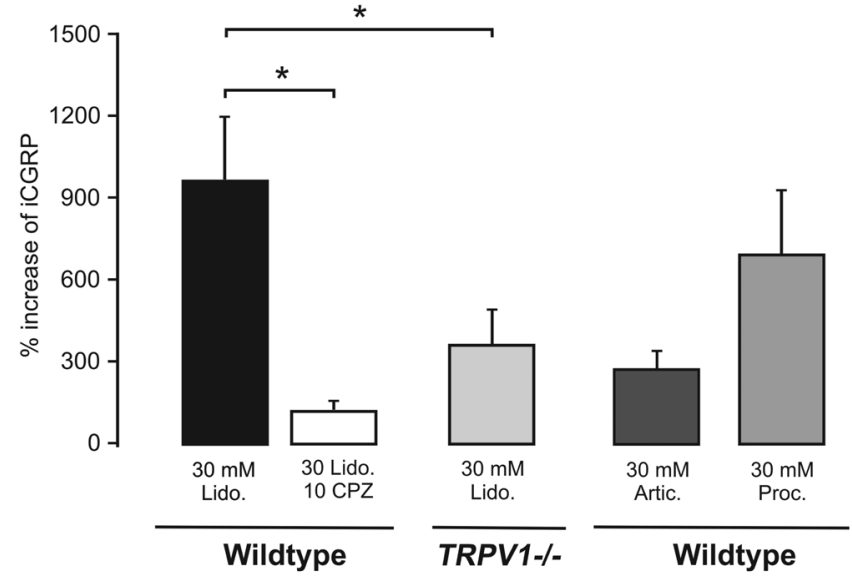

lidocaine, on the other hand, remained capsaicin sensitive both in $\mathrm{Ca}^{2+}$-containing and in $\mathrm{Ca}^{2+}$-free solution. These results clearly demonstrate that lidocaine, capsaicin, and camphor use distinct mechanisms when acting on TRPV1.

A hallmark feature of TRPV1 is its ability to integrate multiple stimuli when simultaneously applied, so that the presence of one agonist promotes the ability of another agonist to induce channel opening (17). Additionally, direct phosphorylation of TRPV1 in a PKC-dependent manner also sensitizes the agonist sensitivity of this channel $(32,33)$. Both effects are reminiscent of the signature changes in nociceptor excitability under inflammation and tissue injury that are mediated by inflammatory mediators that bind to $G$ protein-coupled receptors, including bradykinin and prostaglandins $(42,43)$. We demonstrate here that lidocaine in subthreshold concentrations potentiated TRPV1 currents evoked by thermal stimuli, by capsaicin, or by camphor. Moreover, activation of PKC strongly potentiated lidocaine-activated currents in a manner dependent on intact putative PKC phosphorylation sites. These data suggest that signaling pathways known to be critical for peripheral sensitization under inflammation and tissue injury also might enhance the TRPV1-mediated action of lidocaine on sensory neurons.

We have also shown that activation and sensitization of TRPV1 by LAs required the presence of $\mathrm{PI}(4,5) \mathrm{P}_{2}$ and was abrogated in a mutation within the proximal C-terminal TRP domain, the most conserved cytoplasmic region of TRP channels. The $\mathrm{PI}(4,5) \mathrm{P}_{2}$ interacting site of TRPV1, which might be responsible for its inhibition (30), was not involved in this process, as a mutation

\section{Figure 8}

Lidocaine evokes a TRPV1-dependent release of CGRP in hairy skin and isolated peripheral nerves. (A) Mean increase \pm SEM of CGRP release from hairy skin derived from WT ( $n=8$ per concentration) and TRPV1 ${ }^{-1-}$ mice $(n=8)$. All values were normalized to basal iCGRP that was determined in all preparations before they were treated with different concentrations of lidocaine (1, 10, and $30 \mathrm{mM})$. (B) Mean increase of CGRP release from the sciatic nerve derived from WT and TRPV1-/mice. Values were normalized to basal iCGRP that was determined before treatment with $30 \mathrm{mM}$ lidocaine ( $n=6$ per phenotype), $30 \mathrm{mM}$ lidocaine plus $10 \mu \mathrm{M}$ capsazepine $(n=7), 30 \mathrm{mM}$ articaine $(n=7)$, or $30 \mathrm{mM}$ procaine $(n=7)$ (Wilcoxon test). ${ }^{*} P<0.05$.

lacking the distal $\mathrm{C}$ terminus including this site (TRPV1- $\Delta 767-$ 838) did not affect the ability of lidocaine to activate or sensitize TRPV1. There are several recent reports of $\mathrm{PI}(4,5) \mathrm{P}_{2}$ regulating the activity of members of the TRPV and TRPM subfamilies. In most cases, $\mathrm{PI}(4,5) \mathrm{P}_{2}$ has been found to promote TRP channel activation (44). Accordingly, and in contrast to initial reports, exogenously applied $\mathrm{PI}(4,5) \mathrm{P}_{2}$ was also demonstrated to strongly potentiate, and the $\mathrm{PI}(4,5) \mathrm{P}_{2}$ scavenger poly-L-lysine to suppress, TRPV1 channel activity in excised patches (45). For TRPM8, $\mathrm{PI}(4,5) \mathrm{P}_{2}$ is necessary for activation by cold and menthol, and its depletion leads to $\mathrm{Ca}^{2+}$-dependent desensitization (31). The same study demonstrated that mutations of positively charged residues within the highly conserved proximal C-terminal TRP domain in several TRP channels affect $\mathrm{PI}(4,5) \mathrm{P}_{2}$ interactions, suggesting that $\mathrm{PI}(4,5) \mathrm{P}_{2}$ is a general regulator of TRP channels at this site. Our data indeed suggest that $\mathrm{PI}(4,5) \mathrm{P}_{2}$ might be necessary for activation of TRPV1 by lidocaine as well and that the TRP domain might be involved. However, in our study, 1 of the 3 positively charged residues within the TRP domain that putatively interact with $\mathrm{PI}(4,5) \mathrm{P}_{2}$ in TRPM8 seemed to interact with $\mathrm{PI}(4,5) \mathrm{P}_{2}$ in TRPV1. Mutation of this respective residue in TRPM8 showed the weakest impact on $\mathrm{PI}(4,5) \mathrm{P}_{2}$ interaction in TRPM8 (31).

Our data suggest that PLC activation might be a candidate upstream mechanism of LA-induced $\mathrm{PI}(4,5) \mathrm{P}_{2}$ depletion. Indeed, amphiphilic amine LAs have previously been suggested to increase PLC activity at the cytoplasmic surface of the plasma membrane (37). This could also explain why $\mathrm{Ca}^{2+}$ is not required for lidocaine-induced desensitization, in contrast to menthol-induced desensitization of TRPM8, which is thought to be preceded by $\mathrm{Ca}^{2+}$ influx through the open channels and subsequent PLC activation. A recent study demonstrated that sensitization of TRPA1 by proteinase-activated receptor- 2 also involves PLC and $\mathrm{PI}(4,5) \mathrm{P}_{2}$ signaling (46) and suggests this pathway to be the primary mediator of TRPV1 and TRPA1 activation by LAs.

In summary, it is tempting to hypothesize that $\mathrm{PI}(4,5) \mathrm{P}_{2}$ is required for TRPV1 to be activated by LAs. $\mathrm{PI}(4,5) \mathrm{P}_{2}$ might act as an allosteric modulator, so that in its presence, LAs might be able to activate TRPV1 by interacting with parts of the putative vanilloid binding domain. LAs also increase PLC activity, which leads to $\mathrm{PI}(4,5) \mathrm{P}_{2}$ depletion and subsequent desensitization of TRPV1 toward LAs.

Clinical relevance. The findings of the present study might explain some clinical features of LAs. Pain upon injection of LAs is a source of significant discomfort for patients undergoing LA. One mechanism behind this phenomenon might be the low $\mathrm{pH}$ value of some LA formulations. It is important to note in this context that TRPV1 is considered the main transducing molecule for proton-induced excitation of nociceptive sensory neurons $(14,47)$. The present study 
suggests that LAs themselves might evoke pain upon injection by activating TRPV1. However, LA-evoked pain stops as soon as nerve conduction block sets in. The LA-induced release of neuropeptides as demonstrated in this study might be even more critical in the clinical setting whenever LAs are injected in high concentrations into the skin, to nerves and plexus, or intrathecally. The release of neuropeptides from peripheral and central terminals of sensory neurons induces vascular leakage and dilatation and is thought to contribute to neurogenic inflammation in the periphery and to central sensitization in the spinal dorsal horn (35). Neurogenic inflammation and central sensitization might well underlie the known side effects after spinal anesthesia, transient neurologic symptoms including pain and/or dysesthesia (48). The more severe sequelae after spinal anesthesia, the cauda equina syndrome and lumbosacral neuropathies (49), might be caused by neurotoxic effects of LAs. Several molecular mechanisms have been considered to mediate LA-induced cytotoxicity, including a direct membrane disruption (6), mitochondrial dysfunction (7), and activation of the p38 mitogen-activated protein kinase involved in apoptosis $(8,9)$. It is conceivable that $\mathrm{Ca}^{2+}$ influx as a result of LA-mediated TRPV1 activation contributes to cell death by inducing necrotic and apoptotic mechanisms $(16,50)$. LA-induced cytotoxicity, however, has also been described in several cell systems undoubtedly lacking both TRPV1 and TRPA1. Nevertheless, the data presented here warrant further studies of the role of TRPV1 and TRPA1 in this clinically relevant and fatal process.

\section{Methods}

\begin{abstract}
Animals
Adult Wistar rats (150-200 g), littermates of WT C57BL/6/J mice and TRPV1 $1^{-/-}$mutant mice (25-40 g), were killed by $100 \% \mathrm{CO}_{2}$ (approved by the Animal Protection Authority of the District Government, Regierung von Mittelfranken, Würzburg, Germany). The heterozygous breeding pairs of TRPV1 $1^{-/-}$mice were donated by J.B. Davis (Glaxo-SmithKline, Harlow, UK; ref. 15). They have been continuously backcrossed in our animal facility to C57BL/6/J mice and have been congenic since 5 years. All mice were conventionally genotyped using commercially available primers (Metabion). WT and TRPV1 $1^{-1-}$ knockout alleles were identified with 2 genomic $5^{\prime}$ primers. The sequences were CATGGCCAGTGAGAACACCATGG and AGCCTTTTGTTCTTGGCTTCTCCT for the WT allele and CCGGTGCCCTGAATGAACT and AAGACCGGCTTCCATCCGA for the knockout allele.
\end{abstract}

\section{DRG cell culture}

DRGs were harvested from the lumbar segments and transferred to DMEM (Invitrogen) supplemented with gentamicin $(50 \mu \mathrm{g} / \mathrm{ml}$; SigmaAldrich). DRGs were incubated for $75 \mathrm{~min}$ in collagenase $(0.28 \mathrm{U} / \mathrm{ml}$; Roche Biochemicals) and subsequently for $12 \mathrm{~min}$ in trypsin $(25,000 \mathrm{U} / \mathrm{ml}$; Sigma-Aldrich). After enzymatic digestion, the suspension was dissociated with fire-polished Pasteur pipettes and plated on glass coverslips coated with poly-L-lysine $(200 \mu \mathrm{g} / \mathrm{ml}$; Sigma-Aldrich). DRG neurons were incubated in serum-free TNB 100 medium (Biochrom) supplemented with penicillin and streptomycin $(200 \mathrm{U} / \mathrm{ml}$ each), L-glutamine $(2 \mathrm{mM}$; all from Invitrogen), and nerve growth factor (mouse NGF $2.5 \mathrm{~S}, 100 \mathrm{ng} / \mathrm{ml}$; Alomone Labs) at $37^{\circ} \mathrm{C}$ and $5 \% \mathrm{CO}_{2}$. Electrophysiological recordings or $\mathrm{Ca}^{2+}$ imaging were performed within $24 \mathrm{~h}$ of dissociation.

\section{Mutagenesis and heterologous expression}

Mutagenesis of rTRPV1 and oTRPV1 cDNA was performed as described previously $(26,51)$. All constructs were confirmed by DNA sequencing. HEK293t cells were transfected with WT or mutant plasmids of TRPV1
(0.5-1 or $10 \mu \mathrm{g}$, respectively) or rTRPA1 $(5 \mu \mathrm{g})$ along with a reporter plasmid (CD8-pih3m; $1 \mu \mathrm{g}$ ) by the calcium phosphate precipitation method. After incubation for $12-15 \mathrm{~h}$, the cells were replated in $35-\mathrm{mm}$ culture dishes and used for experiments within 2-3 days. Transfection-positive cells were identified by immunobeads (anti-CD8 Dynabeads; Dynal Biotech).

\section{Ratiometric $\left[\mathrm{Ca}^{2+}\right]_{i}$ measurements}

For $\left[\mathrm{Ca}^{2+}\right]_{\mathrm{i}}$ measurements, neurons were loaded with the fluorescent calcium indicator dye Fura-2 supplied with $0.02 \%$ pluronic F-127 (Invitrogen) for $30 \mathrm{~min}$. Images were acquired with the ImageMaster software package from a peltier-cooled slow-scan CCD camera system with a monochromator (Photon Technology International) coupled to an Axiovert microscope (Zeiss). Fura-2 was excited at $340 \mathrm{~nm}$ and $380 \mathrm{~nm}$ with $200 \mathrm{~ms}$ exposure time at $2 \mathrm{~Hz}$; fluorescence at greater than $420 \mathrm{~nm}$ was collected. Background for both wavelength intensities was continuously recorded and subtracted. Results were reported as a ratio. Cells were continuously superfused with control solution ( $145 \mathrm{mM} \mathrm{NaCl}, 1.25 \mathrm{mM} \mathrm{CaCl}_{2}, 1 \mathrm{mM} \mathrm{MgCl}_{2}$, $5 \mathrm{mM} \mathrm{KCl}, 10 \mathrm{mM}$ glucose, and $10 \mathrm{mM}$ HEPES; adjusted to $\mathrm{pH}$ 7.3). Test solutions were applied through a gravity driven system from a capillary positioned $100-250 \mu \mathrm{m}$ from the cells. All experiments were performed at room temperature. All data were stored on a PC for offline analysis.

\section{Patch clamp recordings}

Whole-cell voltage clamp recordings were performed with small-sized DRG neurons and HEK293t cells. Membrane currents were acquired with an Axopatch 200B amplifier (Molecular Devices), filtered at $1 \mathrm{kHz}$ and sampled at $5 \mathrm{kHz}$. Electrodes were pulled from borosilicate glass tubes (TW150F-3; World Precision Instruments) and heat polished to give a resistance of 1.5-2.0 M $\Omega$. The standard external solution contained $140 \mathrm{mM} \mathrm{NaCl}, 5 \mathrm{mM} \mathrm{KCl}, 2 \mathrm{mM} \mathrm{CaCl} 2,2 \mathrm{mM} \mathrm{MgCl} 2,10 \mathrm{mM}$ HEPES, and $10 \mathrm{mM}$ glucose ( $\mathrm{pH} 7.4$ adjusted with tetramethylammonium hydroxide). In $\mathrm{Ca}^{2+}$-free solutions, $\mathrm{CaCl}_{2}$ was replaced by $5 \mathrm{mM}$ EGTA. The internal solution contained $140 \mathrm{mM} \mathrm{KCl}, 2 \mathrm{mM} \mathrm{MgCl}_{2}, 5 \mathrm{mM}$ EGTA, and $10 \mathrm{mM}$ HEPES ( $\mathrm{pH} 7.4$ adjusted with potassium hydroxide). Unless otherwise noted, cells were held at $-60 \mathrm{mV}$. All recordings were performed at room temperature. Solutions were applied with a polytetrafluoroethylene glass multiple-barrel perfusion system. The heat stimulus was delivered using a multichannel, gravity-driven system incorporating rapid-feedback temperature control. In this system, a platinum-covered glass capillary, positioned less than $100 \mu \mathrm{m}$ from the cell under study, was used as a common outlet (52). The pCLAMP 8.1 software (Axon Instruments) was used for acquisition and offline analysis.

\section{Release of CGRP}

Preparation. The skin from both hind paws of adult mice was subcutaneously dissected below the knee level as described previously (53). The skin flaps obtained $(n=40)$ were tied around acrylic glass rods ( $6-\mathrm{mm}$ diameter) with the corium side exposed. The preparations were washed for $30 \mathrm{~min}$ at $32^{\circ} \mathrm{C}$ in carbogen-gassed $\left(95 \% \mathrm{O}_{2}, 5 \% \mathrm{CO}_{2}\right)$ synthetic interstitial fluid (SIF) containing $108 \mathrm{mM} \mathrm{NaCl}, 3.48 \mathrm{mM} \mathrm{KCl}, 3.5 \mathrm{mM} \mathrm{MgSO}_{4}, 26 \mathrm{mM} \mathrm{NaHCO}_{3}$, $1.7 \mathrm{mM} \mathrm{NaH}_{2} \mathrm{PO}_{4}, 1.5 \mathrm{mM} \mathrm{CaCl}_{2}, 9.6 \mathrm{mM}$ sodium gluconate, $5.5 \mathrm{mM}$ glucose, and $7.6 \mathrm{mM}$ sucrose. Sciatic nerves were excised from where the nerve emerges from the lumbar plexus to the bifurcation into the tibial and peroneal branches. During dissection, the nerve was rinsed continuously with SIF at room temperature. The nerve was loosely tied around an acrylic rod and placed in SIF inside a thermostatic shaking bath at $32^{\circ} \mathrm{C}$.

Stimulation procedures. After a 30-min washout period, skin flaps or nerves were first incubated for $5 \mathrm{~min}$ in test tubes containing control SIF to determine basal CGRP. For stimulation, the preparations were then incubated for $5 \mathrm{~min}$ in tubes containing a test solution. 
Enzyme immunoassay and data analysis. CGRP content of the incubation fluid was measured using commercial enzyme immunoassays (EIAs; SPIbio) immediately after the experiment as described previously in detail (54). All EIA plates were determined photometrically using a microplate reader (Dynatech).

\section{Chemicals}

Lidocaine, procaine, tetracaine, mepivacaine (all from Sigma-Aldrich), articaine (gift from Cilag AG), and QX-314 (Biotrend) were directly dissolved in the extracellular solution. Bupivacaine (Sigma-Aldrich) was dissolved in dimethyl sulfoxide to give a stock solution of $100 \mathrm{mM}$. Unless otherwise noted, the $\mathrm{pH}$ values of all LA solutions were corrected to $\mathrm{pH} 7.4$ with tetramethylammonium hydroxide. Capsaicin, capsazepine, ruthenium red, camphor, and acrolein (all from Sigma-Aldrich) were dissolved in absolute ethanol to give stock solutions of 0.1-1 M. PMA (Calbiochem-Novabiochem GmbH), PAO (Sigma-Aldrich), U73122, U73433, and BCTC (Biotrend) were dissolved in dimethyl sulfoxide to give stock solutions of 1-5 mM.

\section{Statistics}

Calculations for statistical comparisons were performed with the Statistica software package 7.0 (Statsoft) or with the Origin 7.0 software package (OriginLab Corp.). Tests used are denoted in the text and/or figure legends.
A $P$ value less than 0.05 was considered statistically significant. ${ }^{*} P<0.05$; ${ }^{* *} P<0.01 ; * * * P<0.001$. All data are given as mean \pm SEM.

\section{Acknowledgments}

TRPV1 and TRPA1 cDNA were kindly provided by David Julius (UCSF, San Francisco, California, USA). Breeding pairs of TRPV1/mice were kindly provided by John B. Davis (Glaxo-SmithKline, Harlow, United Kingdom). We thank Anne Sterzer, Kerstin Fischer, Nadine Weisel, and Iwona Izydorczyk for excellent technical assistance and J. Schüttler, H. Schwilden, and H.O. Handwerker for continuous support. This work was supported by a grant from the Deutsche Forschungsgemeinschaft (NA-350/3-2, KFO 130) to A. Leffler and C. Nau and by a grant from the European Society of Anesthesiology to C. Nau.

Received for publication May 21, 2007, and accepted in revised form November 7, 2007.

Address correspondence to: Carla Nau, Department of Anesthesiology, Friedrich-Alexander-University of Erlangen-Nuremberg, Krankenhausstrasse 12, 91054 Erlangen, Germany. Phone: 49-9131-85-39154; Fax: 49-9131-85-39161; E-mail: carla.nau@kfa. imed.uni-erlangen.de.
1. Catterall, W.A. 2000. From ionic currents to molecular mechanisms: the structure and function of voltage-gated sodium channels. Neuron. 26:13-25.

2. Nau, C., and Wang, G.K. 2004. Interactions of local anesthetics with voltage-gated $\mathrm{Na}+$ channels. J. Membr. Biol. 201:1-8.

3. Huang, J.H., Thalhammer, J.G., Raymond, S.A., and Strichartz, G.R. 1997. Susceptibility to lidocaine of impulses in different somatosensory afferent fibers of rat sciatic nerve. J. Pharmacol. Exp. Ther. 282:802-811.

4. Nakamura, T., Popitz-Bergez, F., Birknes, J., and Strichartz, G.R. 2003. The critical role of concentration for lidocaine block of peripheral nerve in vivo: studies of function and drug uptake in the rat. Anesthesiology. 99:1189-1197.

5. Hodgson, P.S., Neal, J.M., Pollock, J.E., and Liu, S.S. 1999. The neurotoxicity of drugs given intrathecally (spinal). Anesth. Analg. 88:797-809.

6. Kitagawa, N., Oda, M., and Totoki, T. 2004. Possible mechanism of irreversible nerve injury caused by local anesthetics: detergent properties of local anesthetics and membrane disruption. Anesthesiology. 100:962-967.

7. Johnson, M.E., Uhl, C.B., Spittler, K.H., Wang, H., and Gores, G.J. 2004. Mitochondrial injury and caspase activation by the local anesthetic lidocaine. Anesthesiology. 101:1184-1194.

8. Tan, Z., Dohi, S., Chen, J., Banno, Y., and Nozawa, Y. 2002. Involvement of the mitogen-activated protein kinase family in tetracaine-induced PC12 cell death. Anesthesiology. 96:1191-1201.

9. Lirk, P., et al. 2006. Mitigation of direct neurotoxic effects of lidocaine and amitriptyline by inhibition of p38 mitogen-activated protein kinase in vitro and in vivo. Anesthesiology. 104:1266-1273.

10. Lambert, L.A., Lambert, D.H., and Strichartz, G.R. 1994. Irreversible conduction block in isolated nerve by high concentrations of local anesthetics. Anesthesiology. 80:1082-1093.

11. Gold, M.S., Reichling, D.B., Hampl, K.F., Drasner, K., and Levine, J.D. 1998. Lidocaine toxicity in primary afferent neurons from the rat. J. Pharmacol. Exp. Ther. 285:413-421.

12. Vlachova, V., Vitaskova, Z., Vyklicky, L., and Orkand, R.K. 1999. Procaine excites nociceptors in cultures from dorsal root ganglion of the rat. Neurosci. Lett. 263:49-52.
13. Komai, H., and McDowell, T.S. 2005. Differential effects of bupivacaine and tetracaine on capsaicininduced currents in dorsal root ganglion neurons. Neurosci. Lett. 380:21-25.

14. Caterina, M.J., et al. 2000. Impaired nociception and pain sensation in mice lacking the capsaicin receptor. Science. 288:306-313.

15. Davis, J.B., et al. 2000. Vanilloid receptor-1 is essential for inflammatory thermal hyperalgesia. Nature. 405:183-187.

16. Caterina, M.J., et al. 1997. The capsaicin receptor: a heat-activated ion channel in the pain pathway. Nature. 389:816-824.

17. Tominaga, M., et al. 1998. The cloned capsaicin receptor integrates multiple pain-producing stimuli. Neuron. 21:531-543.

18. Trevisani, M., et al. 2002. Ethanol elicits and potentiates nociceptor responses via the vanilloid receptor-1. Nat. Neurosci. 5:546-551.

19. Xu, H., Blair, N.T., and Clapham, D.E. 2005. Camphor activates and strongly desensitizes the transient receptor potential vanilloid subtype 1 channel in a vanilloid-independent mechanism. J. Neurosci. 25:8924-8937.

20. Ahern, G.P., Brooks, I.M., Miyares, R.L., and Wang, X.B. 2005. Extracellular cations sensitize and gate capsaicin receptor TRPV1 modulating pain signaling. J. Neurosci. 25:5109-5116.

21. Ahern, G.P., Wang, X., and Miyares, R.L. 2006. Polyamines are potent ligands for the capsaicin receptor TRPV1. J. Biol. Chem. 281:8991-8995.

22. Vellani, V., Mapplebeck, S., Moriondo, A., Davis, J.B., and McNaughton, P.A. 2001. Protein kinase $\mathrm{C}$ activation potentiates gating of the vanilloid receptor VR1 by capsaicin, protons, heat and anandamide. J. Physiol. 534:813-825.

23. Bautista, D.M., et al. 2006. TRPA1 mediates the inflammatory actions of environmental irritants and proalgesic agents. Cell. 124:1269-1282.

24. Jordt, S.E., Tominaga, M., and Julius, D. 2000. Acid potentiation of the capsaicin receptor determined by a key extracellular site. Proc. Natl. Acad. Sci.U. S. A. 97:8134-8139.

25. Jordt, S.E., and Julius, D. 2002. Molecular basis for species-specific sensitivity to "hot" chili peppers. Cell. 108:421-430.

26. Gavva, N.R., et al. 2004. Molecular determinants of vanilloid sensitivity in TRPV1. J. Biol. Chem. 279:20283-20295.

27. Numazaki, M., et al. 2003. Structural determinant of TRPV1 desensitization interacts with calmodulin. Proc. Natl. Acad. Sci. U. S. A. 100:8002-8006.

28. Rosenbaum, T., Gordon-Shaag, A., Munari, M., and Gordon, S.E. 2004. Ca2+/calmodulin modulates TRPV1 activation by capsaicin. J. Gen. Physiol. 123:53-62.

29. Mohapatra, D.P., and Nau, C. 2003. Desensitization of capsaicin-activated currents in the vanilloid receptor TRPV1 is decreased by the cyclic AMPdependent protein kinase pathway. J. Biol. Chem. 278:50080-50090.

30. Prescott, E.D., and Julius, D. 2003. A modular PIP2 binding site as a determinant of capsaicin receptor sensitivity. Science. 300:1284-1288.

31. Rohacs, T., Lopes, C.M., Michailidis, I., and Logothetis, D.E. 2005. $\mathrm{PI}(4,5) \mathrm{P} 2$ regulates the activation and desensitization of TRPM8 channels through the TRP domain. Nat. Neurosici. 8:626-634.

32. Numazaki, M., Tominaga, T., Toyooka, H., and Tominaga, M. 2002. Direct phosphorylation of capsaicin receptor VR1 by protein kinase Cepsilon and identification of two target serine residues. J. Biol. Chem. 277:13375-13378.

33. Bhave, G., et al. 2003. Protein kinase C phosphorylation sensitizes but does not activate the capsaicin receptor transient receptor potential vanilloid 1 (TRPV1). Proc. Natl. Acad. Sci. U. S. A. 100:12480-12485.

34. Fischer, M.J., Reeh, P.W., and Sauer, S.K. 2003. Proton-induced calcitonin gene-related peptide release from rat sciatic nerve axons, in vitro, involving TRPV1. Eur. J. Neurosci. 18:803-810.

35. Planells-Cases, R., Garcia-Sanz, N., MorenillaPalao, C., and Ferrer-Montiel, A. 2005. Functional aspects and mechanisms of TRPV1 involvement in neurogenic inflammation that leads to thermal hyperalgesia. Pflugers Arch. 451:151-159.

36. Mogil, J.S., et al. 2005. Variable sensitivity to noxious heat is mediated by differential expression of the CGRP gene. Proc. Natl. Acad. Sci. U. S. A. 102:12938-12943.

37. Raucher, D., and Sheetz, M.P. 2001. Phospholipase $\mathrm{C}$ activation by anesthetics decreases membranecytoskeleton adhesion. J. Cell Sci. 114:3759-3766. 
38. Mohapatra, D.P., and Nau, C. 2005. Regulation of $\mathrm{Ca} 2+$-dependent desensitization in the vanilloid receptor TRPV1 by calcineurin and cAMP-dependent protein kinase. J. Biol. Chem. 280:13424-13432.

39. Bhave, G., et al. 2002. cAMP-dependent protein kinase regulates desensitization of the capsaicin receptor (VR1) by direct phosphorylation. Neuron. 35:721-731.

40. Mandadi, S., et al. 2004. Activation of protein kinase $C$ reverses capsaicin-induced calciumdependent desensitization of TRPV1 ion channels. Cell Calcium. 35:471-478.

41. Liu, B., Zhang, C., and Qin, F. 2005. Functional recovery from desensitization of vanilloid receptor TRPV1 requires resynthesis of phosphatidylinositol 4,5-bisphosphate. J. Neurosci. 25:4835-4843.

42. Chuang, H.H., et al. 2001. Bradykinin and nerve growth factor release the capsaicin receptor from PtdIns(4,5)P2-mediated inhibition. Nature. 411:957-962.

43. Moriyama, T., et al. 2005. Sensitization of TRPV1 by EP1 and IP reveals peripheral nociceptive mech- anism of prostaglandins. Mol. Pain. 1:3.

44. Hardie, R.C. 2007. TRP channels and lipids: from Drosophila to mammalian physiology. J. Physiol. 578:9-24.

45. Stein, A.T., Ufret-Vincenty, C.A., Hua, L., Santana, L.F., and Gordon, S.E. 2006. Phosphoinositide 3kinase binds to TRPV1 and mediates NGF-stimulated TRPV1 trafficking to the plasma membrane. J. Gen. Physiol. 128:509-522.

46. Dai, Y., et al. 2007. Sensitization of TRPA1 by PAR2 contributes to the sensation of inflammatory pain. J. Clin. Invest. 117:1979-1987.

47. Leffler, A., Monter, B., and Koltzenburg, M. 2006 The role of the capsaicin receptor TRPV1 and acidsensing ion channels (ASICS) in proton sensitivity of subpopulations of primary nociceptive neurons in rats and mice. Neuroscience. 139:699-709.

48. Pollock, J.E. 2002. Transient neurologic symptoms: etiology, risk factors, and management. Reg. Anesth. Pain Med. 27:581-586.

49. Rigler, M.L., et al. 1991. Cauda equina syndrome after continuous spinal anesthesia. Anesth. Analg.
72:275-281.

50. Kim, S.R., et al. 2005. Transient receptor potential vanilloid subtype 1 mediates cell death of mesencephalic dopaminergic neurons in vivo and in vitro. J. Neurosci. 25:662-671.

51. Mohapatra, D.P., Wang, S.Y., Wang, G.K., and Nau, C. 2003. A tyrosine residue in TM6 of the Vanilloid Receptor TRPV1 involved in desensitization and calcium permeability of capsaicin-activated currents. Mol. Cell. Neurosci. 23:314-324.

52. Dittert, I., et al. 2006. Improved superfusion technique for rapid cooling or heating of cultured cells under patch-clamp conditions. J. Neurosci. Methods. 151:178-185.

53. Sauer, S.K., Schafer, D., Kress, M., and Reeh, P.W. 1998. Stimulated prostaglandin E2 release from rat skin, in vitro. Life Sci. 62:2045-2055.

54. Kress, M., Guthmann, C., Averbeck, B., and Reeh, P.W. 1999. Calcitonin gene-related peptide and prostaglandin E2 but not substance P release induced by antidromic nerve stimulation from rat skin in vitro. Neuroscience. 89:303-310. 